\title{
ON VANISHING OF KRONECKER COEFFICIENTS
}

\author{
Christian Ikenmeyer, Ketan D. Mulmuley, \\ AND MiCHAEL WALTER \\ July 24, 2017
}

\begin{abstract}
We show that the problem of deciding positivity of Kronecker coefficients is NP-hard. Previously, this problem was conjectured to be in $\mathrm{P}$, just as for the Littlewood-Richardson coefficients. Our result establishes in a formal way that Kronecker coefficients are more difficult than Littlewood-Richardson coefficients, unless $\mathrm{P}=\mathrm{NP}$.

We also show that there exists a \#P-formula for a particular subclass of Kronecker coefficients whose positivity is NP-hard to decide. This is an evidence that, despite the hardness of the positivity problem, there may well exist a positive combinatorial formula for the Kronecker coefficients. Finding such a formula is a major open problem in representation theory and algebraic combinatorics.
\end{abstract}

Finally, we consider the existence of the partition triples $(\lambda, \mu, \pi)$ such that the Kronecker coefficient $k_{\mu, \pi}^{\lambda}=0$ but the Kronecker coefficient $k_{l \mu, l \pi}^{l \lambda}>0$ for some integer $l>1$. Such "holes" are of great interest as they witness the failure of the saturation property for the Kronecker coefficients, which is still poorly understood. Using insight from computational complexity theory, we turn our hardness proof into a positive result: We show that not only do there exist many such triples, but they can also be found efficiently. Specifically, we show that, for any $0<\epsilon \leq 1$, there exists $0<a<1$ such that, for all $m$, there exist $\Omega\left(2^{m^{a}}\right)$ partition triples $(\lambda, \mu, \mu)$ in the Kronecker cone such that: (a) the Kronecker coefficient $k_{\mu, \mu}^{\lambda}$ is zero, (b) the height of $\mu$ is $m$, (c) the height of $\lambda$ is $\leq m^{\epsilon}$, and (d) $|\lambda|=|\mu| \leq m^{3}$. The proof of the last result illustrates the effectiveness of the explicit proof strategy of GCT.

Keywords. Geometric complexity theory, Kronecker coefficients, Moment polytope, Computational Complexity, NP-hard. 
Subject classification. 68Q17, 05E10.

\section{Introduction}

One class of representation-theoretic obstructions in the context of the geometric complexity theory (GCT) approach to the permanent vs. determinant problem (Mulmuley 2011; Mulmuley \& Sohoni 2008) is based on the existence of vanishing rectangular Kronecker coefficients (Bürgisser et al. 2011a; Kumar 2015; Mulmuley \& Sohoni 2008). These are called occurrence-based obstructions, as opposed to the more general multiplicity-based obstructions. We refer to Bürgisser et al. (2011b); Bürgisser (2016) for introduction and background. It is now known that such occurrence-based obstructions based on vanishing of Kronecker coefficients cannot be used for proving superpolynomial lower bounds for the permanent (Ikenmeyer \& Panova 2016). However, they may still be useful for proving modest polynomial lower bounds. The partition triples associated with the rectangular Kronecker coefficients lie in the moment cone (Kirwan 1984) associated with the Kronecker coefficients, called the Kronecker cone (Bürgisser et al. 2011a; Kumar 2015). As pointed out in Kumar (2015), this makes the problem of showing the existence of such partition triples rather challenging, since the asymptotic techniques of algebraic geometry and representation theory, such as the ones based on the effective descriptions of the linear inequalities defining the Kronecker cone (Berenstein \& Sjamaar 2000; Klyachko 2004; Ressayre 2010; Vergne \& Walter 2017), cannot be used to prove this existence.

The main result in this article (Theorem 1.7) establishes the existence of a superpolynomial number of partition triples with vanishing Kronecker coefficients, in the Kronecker cone for the given partition size, and satisfying a relaxed form of the additional shape restrictions that arise in GCT.

Its proof, based on the explicit proof strategy of GCT (Mulmuley 2010a b, 2011), also yields results concerning the complexity of Kronecker coefficients that are of independent interest. The first such result (Theorem 1.3) shows that the problem of deciding positivity of Kronecker coefficients is NP-hard. The second result 
Theorem 1.5 gives the first known instance of a positive (\#P) formula for a subclass of Kronecker coefficients whose positivity is NP-hard to decide.

We now state these results in more detail after the following preliminary section.

\subsection{Preliminaries in algebraic combinatorics and repre-} sentation theory. An partition $\lambda$ is defined to be a finite nonincreasing sequence of positive integers $\lambda=\left(\lambda_{1}, \lambda_{2}, \ldots, \lambda_{\ell}\right)$. We say that $\lambda$ has $\ell$ nonzero parts and define the height ht $(\lambda)$ of $\lambda$ to be $\ell$. We define $\lambda_{i}:=0$ for all $i>\ell$ and set $|\lambda|:=\sum_{i} \lambda_{i}$. To each partition we associate a so-called Young diagram, which is a left-top-justified array of boxes in which the $i$ th row contains exactly $\lambda_{i}$ boxes. For example, the Young diagram to the partition $(3,1)$ is $\square \square$. We often identify Young diagrams with their partitions and say that $\lambda$ has $|\lambda|$ many boxes. Transposing a Young diagram at the main diagonal gives another Young diagram and we call the corresponding partition the transpose partition of $\lambda$, denoted by $\lambda^{T}$. For example, $(3,1)^{T}=(2,1,1)$, because transposing $(3,1)$ gives the Young diagram $\square$.

When we encode partitions as bit strings there are two fundamentally different ways of doing it: As a list of numbers in binary or as a list of numbers in unary. Note that in unary transposing a partition does not significantly change its encoding size, but in binary the 1-row partition $(n)$ can be encoded using $O(\log n)$ bits, while $(n)^{T}=(1,1, \ldots, 1)$ requires $O(n)$ bits. We will mostly encode partitions in unary.

It is natural to interpret partitions as vectors with integer entries, so that we have a well-defined addition and scalar multiplication with nonnegative integers. Moreover, dividing a partition by an integer results in a vector with rational entries.

Let $G:=\mathrm{GL}_{r}$ denote the general linear group, i.e., the group of invertible $r \times r$ matrices. Let $V$ be a finite dimensional vector space and let $\mathrm{GL}(V)$ denote the set of linear isomorphisms of $V$. A group homomorphism $\varrho: G \rightarrow \mathrm{GL}(V)$ is called a representation of $G$. We say that $V$ is a representation if $\varrho$ is clear from the context. We say that $G$ acts linearly on $V$ and use the short notation $g v:=(\varrho(g))(v)$ 
for $g \in G, v \in V$. If all the coordinate functions of $\varrho$ are given by multivariate polynomials in the $r^{2}$ coordinate variables of $\mathrm{GL}_{r}$, then we call $\varrho$ a polynomial representation.

A linear subspace $W \subseteq V$ that satisfies $\forall w \in W, g \in G: g w \in$ $W$, is called a subrepresentation. Subrepresentations of polynomial representations are always polynomial. For every representation $V$, the zero vector space and $V$ itself are two subrepresentations. If $V$ has only these two subrepresentations, then $V$ is called irreducible. Given two representations $\left(V, \rho_{V}\right)$ and $\left(W, \rho_{W}\right)$, then a linear map $\varphi: V \rightarrow W$ is called equivariant if $g \varphi(v)=\varphi(g v)$ for all $g \in G$, $v \in V$. If $\varphi$ is an equivariant isomorphism of vector spaces, then $\varphi$ is called a $G$-isomorphism and the representations $V$ and $W$ are called isomorphic. The different types of isomorphic irreducible polynomial representations of $G$ have been classified completely: They are indexed by partitions of height at most $r$. In a representation $V$ the sum of all subrepresentations of type $\lambda$ is called the $\lambda$-isotypic component. Every representation decomposes into a direct sum of isotypic components.

A vector $v \in V$ that is rescaled by diagonal matrices is called a weight vector, i.e., if $\operatorname{diag}\left(\alpha_{1}, \ldots, \alpha_{r}\right) v=\alpha_{1}^{\lambda_{1}} \cdots \alpha_{r}^{\lambda_{r}} v$, then $v$ is called a weight vector of weight $\lambda$. For this definition it is not necessary that $\lambda$ is nonincreasing. The weight vectors of weight $\lambda$ form a vector space which is called the weight subspace of $V$ of weight $\lambda$. A representation decomposes into a direct sum of weight spaces. In each irreducible representation of type $\lambda$ there is a unique line of weight $\lambda$. This line is called the highest weight line. The highest weight line is also characterized by the Lie algebra action as follows. For a representation $V$ let $v \in V$ and $A \in \mathfrak{g}:=\mathbb{C}^{r \times r}$. Note that $\varepsilon A+\operatorname{Id}_{r} \in G$ for small $\varepsilon$, where $\operatorname{Id}_{r}$ is the $r \times r$ identity matrix. The Lie algebra action of $A$ on $v$ is defined as $A . v:=\lim _{\varepsilon \rightarrow 0} \varepsilon^{-1}((\varepsilon A+\mathrm{Id}) v-v)$. Clearly $A . v \in V$. If we pick $A=E_{i, j}$ to be the matrix that has a single 1 at position $(i, j)$ with $i<j$ and zeros everywhere else, then the map $v \mapsto E_{i, j} . v$ is called a raising operator. A weight vector that is mapped to zero by all raising operators is called a highest weight vector. Each irreducible representation has a unique line of highest weight vectors and their weight determines the type of the irreducible representation. 
The multiplicity of the type $\lambda$ in a representation $V$ is the dimension of the vector space of highest weight vectors of type $\lambda$. If we decompose $V$ into a direct sum of irreducibles, then this multiplicity counts how often a copy of type $\lambda$ appears in the decomposition.

If we have $k$ commuting actions of several copies of $G$ on $V$, then we use the representation theory of the cartesian powers $G^{k}$, which is very similar to the representation theory of $G$. We will mainly be concerned with $k=2$ and $k=3$. The types of irreducible representations of $G^{k}$ are given by $k$-tuples of partitions, weight vectors are defined by their scaling behavior under $k$-tuples of diagonal matrices, and the Lie algebra action is defined via $k$-tuples of matrices, where the raising operators are $k$-tuples of matrices in which only one matrix is nonzero. Irreducible representations of $G$ are called Weyl modules, while irreducible representations of $G^{k}$ are isomorphic to a $k$-fold tensor product of Weyl modules.

Using the group homomorphism $\mathrm{GL}_{r} \times \mathrm{GL}_{r} \rightarrow \mathrm{GL}_{r^{2}},\left(g, g^{\prime}\right) \mapsto$ $g \otimes g^{\prime}$, every representation of $\mathrm{GL}_{r^{2}}$ is also a representation of $\mathrm{GL}_{r} \times \mathrm{GL}_{r}$. For a partition $\lambda$ let $V_{\lambda}\left(\mathrm{GL}_{r^{2}}\right)$ be the Weyl module of type $\lambda$. Even though $V_{\lambda}\left(\mathrm{GL}_{r^{2}}\right)$ is an irreducible $\mathrm{GL}_{r^{2}}$ representation, $V_{\lambda}\left(\mathrm{GL}_{r^{2}}\right)$ decomposes into a nontrivial direct sum of isotypic components with respect to the action of $\mathrm{GL}_{r} \times \mathrm{GL}_{r}$. For partitions $\mu$ and $\pi$ the multiplicity of the irreducible $V_{\mu}\left(\mathrm{GL}_{r}\right) \otimes V_{\pi}\left(\mathrm{GL}_{r}\right)$ in $V_{\lambda}\left(\mathrm{GL}_{r^{2}}\right)$ is called the Kronecker coefficient $k_{\mu, \pi}^{\lambda} \in \mathbb{N}$ (Fulton \& Harris 1991). If $\operatorname{ht}(\lambda) \leq r^{2}, \operatorname{ht}(\mu) \leq r$, and $\operatorname{ht}(\pi) \leq r$, then $k_{\mu, \pi}^{\lambda}$ does not depend on $r$. Otherwise $k_{\mu, \pi}^{\lambda}=0$.

The Kronecker coefficient arises as a multiplicity in several other representation theoretic decompositions, for example as the multiplicity of $V_{\lambda}\left(\mathrm{GL}_{r}\right) \otimes V_{\mu}\left(\mathrm{GL}_{r}\right) \otimes V_{\pi}\left(\mathrm{GL}_{r}\right)$ in $V_{(n)}\left(\mathrm{GL}_{r^{3}}\right)$ via the group homomorphism $\mathrm{GL}_{r}^{3} \rightarrow \mathrm{GL}_{r^{3}},\left(g, g^{\prime}, g^{\prime \prime}\right) \mapsto g \otimes g^{\prime} \otimes g^{\prime \prime}$, where $\lambda, \mu$, and $\pi$ have exactly $n$ boxes.

For $k_{\mu, \pi}^{\lambda}$ to be positive it is required that $\lambda, \mu$, and $\pi$ are partitions of the same number, i.e., $|\lambda|=|\mu|=|\pi|=n$ for some $n$. This implies that if $k_{\mu, \pi}^{\lambda}>0$, then the rescaled partitions $\lambda / n$, $\mu / n$, and $\pi / n$ are three discrete probability distributions. Another necessary condition for $k_{\mu, \pi}^{\lambda}>0$ is $\operatorname{ht}(\lambda) \leq \operatorname{ht}(\mu) \cdot \operatorname{ht}(\pi)$. The 
coefficient is invariant under permuting the three parameters, so

$$
k_{\mu, \pi}^{\lambda}=k_{\pi, \mu}^{\lambda}=k_{\pi, \lambda}^{\mu}
$$

Moreover, transposing any two of the three parameters does not change the coefficient:

$$
k_{\mu, \pi}^{\lambda}=k_{\mu^{T}, \pi^{T}}^{\lambda}
$$

Another important description of the Kronecker coefficient is presented in Lemma 2.1. The tensor power $\otimes^{3} \mathbb{C}^{r}=\left(\mathbb{C}^{r}\right)^{\otimes 3}$ has a canonical action of $G^{3}$ via $\left(g, g^{\prime}, g^{\prime \prime}\right)(u \otimes v \otimes w)=(g u) \otimes\left(g^{\prime} v\right) \otimes$ $\left(g^{\prime \prime} w\right)$ for all $\left(g, g^{\prime}, g^{\prime \prime}\right) \in G^{3}$ and $u \otimes v \otimes w$ a rank 1 tensor from $\left(\mathbb{C}^{r}\right)^{\otimes 3}$, where the action is defined by linear continuation. This action induces an action on the $n$-th tensor power $\otimes^{n}\left(\left(\mathbb{C}^{r}\right)^{\otimes 3}\right)$ via $\left(g, g^{\prime}, g^{\prime \prime}\right)\left(\left(u_{1} \otimes v_{1} \otimes w_{1}\right) \otimes\left(u_{2} \otimes v_{2} \otimes w_{2}\right) \otimes \cdots \otimes\left(u_{n} \otimes v_{n} \otimes w_{n}\right)\right):=$ $\left(\left(g u_{1} \otimes g^{\prime} v_{1} \otimes g^{\prime \prime} w_{1}\right) \otimes\left(g u_{2} \otimes g^{\prime} v_{2} \otimes g^{\prime \prime} w_{2}\right) \otimes \cdots \otimes\left(g u_{n} \otimes g^{\prime} v_{n} \otimes g^{\prime \prime} w_{n}\right)\right)$. The antisymmetric tensors form a subrepresentation: $\bigwedge^{n}\left(\mathbb{C}^{r}\right)^{\otimes 3} \subseteq$ $\otimes^{n}\left(\mathbb{C}^{r}\right)^{\otimes 3}$. The Kronecker coefficient $k_{\mu, \pi}^{\lambda}$ is equal to the multiplicity of the irreducible representation $V_{\lambda^{T}}\left(\mathrm{GL}_{r}\right) \otimes V_{\mu^{T}}\left(\mathrm{GL}_{r}\right) \otimes V_{\pi^{T}}\left(\mathrm{GL}_{r}\right)$ of $\mathrm{GL}_{r}^{3}$ in $\bigwedge^{n}\left(\mathbb{C}^{r}\right)^{\otimes 3}$, provided $r$ is large enough.

Given two partition triples $(\lambda, \mu, \pi)$ and $\left(\lambda^{\prime}, \mu^{\prime}, \pi^{\prime}\right)$ such that $k_{\mu, \pi}^{\lambda}>0$ and $k_{\mu^{\prime}, \pi^{\prime}}^{\lambda^{\prime}}>0$, then $k_{\mu+\mu^{\prime}, \pi+\pi^{\prime}}^{\lambda+\lambda^{\prime}}>0$. This is called the semigroup property. The convex cone defined by

$$
\operatorname{Kron}(r):=\operatorname{cone}\left\{(\lambda, \mu, \pi) \mid \operatorname{ht}(\lambda), \operatorname{ht}(\mu), \operatorname{ht}(\pi) \leq r, k_{\mu, \pi}^{\lambda}>0\right\}
$$

is a polyhedral cone (Kirwan 1984), called the Kronecker cone. Here we think of $(\lambda, \mu, \pi)$ as a vector in $\mathbb{Q}^{3 r}$. A partition triple outside of this cone trivially has a zero Kronecker coefficient.

Finding a combinatorial description of $k_{\mu, \pi}^{\lambda}$ is an important outstanding problem (see Section 1.3 below). Only for some special cases is a combinatorial description known, for example for the Littlewood-Richardson coefficients. The Littlewood-Richardson coefficients are those $k_{\mu, \pi}^{\lambda}$ for which $\lambda, \mu$, and $\pi$ have a sufficiently long first row such that $|\bar{\lambda}|=|\bar{\mu}|+|\bar{\pi}|$, where $\bar{\lambda}$ is the partition $\lambda$ with its longest row removed. The positivity of Littlewood-Richardson coefficients can be decided in strongly polynomial time (Knutson \& Tao 2001; Mulmuley et al. 2011). 
Another important subclass of Kronecker coefficients are the rectangular Kronecker coefficients. For a given partition $\lambda$ with size $|\lambda|$ divisible by $r$, let $\delta(\lambda)$ denote the rectangular partition $(d, \ldots, d)$ ( $r$ times), where $d=|\lambda| / r$. We call the Kronecker coefficient $k_{\delta(\lambda), \delta(\lambda)}^{\lambda}$ rectangular. Rectangular Kronecker coefficients play a special role in geometric complexity theory (see Section 1.4 below).

\subsection{NP-hardness of deciding positivity of Kronecker coef-} ficients. Let KRONECKER be the problem of deciding positivity of $k_{\mu, \pi}^{\lambda}$, given as input the three partitions $\lambda, \mu$, and $\pi$ in unary. This problem is of fundamental interest in the context of the explicit proof strategy of GCT (Mulmuley 2010a b, 2011). Our first result is the following:

\section{THEOREM 1.3. KRONECKER is NP-hard.}

It was conjectured in Mulmuley (2010b) that the problem of deciding positivity of Kronecker coefficients is in $P$. Theorem 1.3 shows that this is not so, in general, assuming that $P \neq N P$. This is in contrast to the special case of the Littlewood-Richardson coefficients, where positivity can be decided in strongly polynomial time, as explained above.

\subsection{A \#P-formula for a subclass of partitions of type NP.}

To find a positive formula for Kronecker coefficients "akin to" the well known positive Littlewood-Richardson rule is an unsolved problem in classical representation theory. We refer to Stanley (2002) for the history and importance of this problem, where it is listed as one of the twenty-five "outstanding open problems". In classical representation theory, the phrase "akin to" is used only informally. A formal complexity-theoretic version of this problem is to find a \#P-formula for Kronecker coefficients. By a \#P-formula for the Kronecker coefficient $k_{\mu, \pi}^{\lambda}$, we mean a formula of the form:

$$
k_{\mu, \pi}^{\lambda}=\sum_{\sigma \in\{0,1\}^{p(\langle\lambda\rangle,\langle\mu\rangle,\langle\pi\rangle)}} F(\lambda, \mu, \pi, \sigma),
$$

where, for a partition $\lambda=\left(\lambda_{1}, \lambda_{2}, \ldots, \lambda_{l}\right),\langle\lambda\rangle$ denotes the total bitlength of the specification of $\lambda_{j}$ 's in binary, $p(\langle\lambda\rangle,\langle\mu\rangle,\langle\pi\rangle)$ is a 
polynomially-bounded function of the bit-lengths $\langle\lambda\rangle,\langle\mu\rangle$, and $\langle\pi\rangle$, and $F(\lambda, \mu, \pi, \sigma)$ is a polynomial-time-computable $0-1$ function of $\lambda, \mu, \pi$, and the bit-string $\sigma$. By a positive formula, we mean a \#P-formula henceforth.

Definition 1.4. Let $\Pi$ be a class of partition triples. We say that $\Pi$ is of type NP if the problem of deciding positivity of $k_{\mu, \pi}^{\lambda}$, with $(\lambda, \mu, \pi) \in \Pi$, is NP-hard. (The problem mentioned here is a promise problem. That is, we are promised that the input triple is in the subclass $\Pi$.) Likewise, we say that $\Pi$ is of type $\mathrm{P}$ if the problem of deciding positivity of $k_{\mu, \pi}^{\lambda}$, with $(\lambda, \mu, \pi) \in \Pi$, is in $P$.

All positive rules known so far for restricted classes of Kronecker coefficients have been for subclasses of partition triples that are either known or conjectured to be of type P. For example, the classical Littlewood-Richardson rule gives a positive rule for Littlewood-Richardson coefficients, which, as already mentioned, constitute a special class of Kronecker coefficients. The corresponding subclass of partition triples is of type $\mathrm{P}$, since the problem of deciding positivity of Littlewood-Richardson coefficients is in $P$ (Knutson \& Tao 2001; Mulmuley et al. 2011). Blasiak et al. (2015) give a positive rule for Kronecker coefficients when two of the partitions have height at most two. The corresponding subclass of partition triples is of type $\mathrm{P}$, since the Kronecker coefficient can be computed in this case (and more generally, for partitions of bounded height) in polynomial time (Christandl et al. (2012); Baldoni et al. (2017)). Blasiak (2017) gives a positive rule for Kronecker coefficients when one of the partitions is a hook. The corresponding subclass of partition triples is conjectured to be of type $\mathrm{P}$, since the problem of deciding positivity of Kronecker coefficients, when one of the partitions is a hook, is believed to be in $P$ (in view of Theorem 6.6).

The following result gives the first known instance of a positive rule for Kronecker coefficients for a subclass of partition triples of type NP.

TheOREM 1.5. There exists a \#P-formula for Kronecker coefficients for a subclass of partition triples of type NP. Here the partition triples can be specified in unary or binary. 
The proof of this result exhibits an explicit such subclass of partition triples of type NP (see Section 2 and Section 3).

Theorem 1.5 provides good evidence in support of the conjecture in Mulmuley (2010b) that there exists a \#P-formula for Kronecker coefficients in general. This would in particular imply that KRONECKER is in NP, which is not known so far.

1.4. Exceptional Kronecker coefficients. In order for a Kronecker coefficient $k_{\mu, \pi}^{\lambda}$ to be useful for proving a polynomial lower bound for the permanent, the partition triple must have a number of exceptional properties (Bürgisser et al. 2011b; Mulmuley \& Sohoni 2008). This is captured by the following definition:

Definition 1.6. Fix any constant $0<\epsilon \leq 1$, and a constant $b>1$. We call a partition triple $(\lambda, \mu, \pi)$ with $|\lambda|=|\mu|=|\pi|$ $(\epsilon, b)$-exceptional if:

(i) $k_{\mu, \pi}^{\lambda}=0$,

(ii) $\mu=\pi=\delta(\lambda)$, with $|\lambda|=|\mu|=|\pi|$ divisible by $r:=\operatorname{ht}(\mu)=$ $\operatorname{ht}(\pi)$,

(iii) $\operatorname{ht}(\lambda) \leq r^{\epsilon}$,

(iv) $(\lambda, \mu, \pi) \in \operatorname{Kron}(r)$,

(v) $|\lambda|=|\mu|=|\pi| \leq r^{b}$,

(vi) the multiplicity $p(\lambda)$ of the Weyl module $V_{\lambda}\left(G L_{r^{2}}(\mathbb{C})\right)$ in $\operatorname{Sym}^{d}\left(\operatorname{Sym}^{r}\left(\mathbb{C}^{r^{2}}\right)\right), d=|\lambda| / r$, is positive, and

(vii) $\lambda_{0} \geq|\lambda|\left(1-r^{\epsilon / 2-1}\right)$.

We also call a partition tuple merely exceptional, without mentioning $\epsilon$ and $b$, if it is understood that $\epsilon$ can be chosen to be arbitrarily small, with $b$ a large enough constant depending on $\epsilon$, and $r \rightarrow \infty$.

By Bürgisser et al. (2011a), (ii) implies (iv), assuming that the height of $\lambda$ is $\leq r^{2}$, which is so by (iii).

The constraint (iv) is significant. Proving existence of the partition triples as in Definition 1.6 is delicate because of this constraint. 
Indeed, it may be possible to prove existence of superpolynomially many partition triples satisfying the constraints other than (ii) and (iv) using the known linear inequalities defining the Kronecker cone (Berenstein \& Sjamaar 2000; Klyachko 2004; Ressayre 2010; Vergne \& Walter 2017). But the constraint (iv) implies that such asymptotic techniques based on the description of the Kronecker cone cannot be used to demonstrate existence of partition triples as in Definition 1.6. This is the main significance of the results in Bürgisser et al. (2011a); Kumar (2015).

By the Saturation Theorem (Derksen \& Weyman 2000; Knutson \& Tao 1999), the Littlewood-Richardson coefficients cannot vanish for the partition triples that lie in the analogously defined Littlewood-Richardson cone. The constraint (iv) also implies that in order to prove existence of the partition triples as in Definition 1.6. one needs to understand the failure of the saturation property for the Kronecker coefficients in one way or another.

The constraint (vii) is motivated by Kadish \& Landsberg (2014). There, it is shown that this condition holds if $V_{\lambda}(G)$ is a representation-theoretic obstruction (Mulmuley \& Sohoni 2008).

It is a priori not at all clear that for any given constant $0<\epsilon \leq 1$ and a large enough constant $b>1$ depending on $\epsilon$, exceptional partition triples exist for arbitrary $r$. The experimental evidence in Ikenmeyer (2012) for small values of $r$ (with suitable $\epsilon$ and $b$ ) suggests that they are very rare, though they do exist for these small values. In summary, although their density can be expected to be extremely small, it is a relevant and rather non-trivial problem in the context of GCT to show that exceptional partition triples exist and that their number is large enough.

\subsection{Construction of superpolynomially many partition tri- ples in the Kronecker cone with vanishing Kronecker co-} efficients. As the first step towards this goal, we relax the condition (ii) to the weaker requirement that only $\mu=\pi$, the condition (vi) to the weaker requirement weaker that $\lambda$ is not a hook (since it can be shown that $p(\lambda)=0$ if $\lambda$ is a hook), and ignore the condition (vii). A priori, it is not clear that partition triples with these properties exist even after this shape relaxation, since condition (iv) is retained. The following result shows that the number 
of Kronecker coefficients with this relaxation of Definition 1.6 is superpolynomial.

TheOrem 1.7 (The main result). For any $0<\epsilon \leq 1$, there exists $0<a<1$, such that, for all $m$, there exist $\Omega\left(2^{m^{a}}\right)$ partition triples $(\lambda, \mu, \pi)$ such that

(i) $k_{\mu, \pi}^{\lambda}=0$,

(ii) $\mu=\pi$,

(iii) $\operatorname{ht}(\mu)=m$, and $\mathrm{ht}(\lambda) \leq m^{\epsilon}$,

(iv) $(\lambda, \mu, \pi) \in \operatorname{Kron}(m)$,

(v) $|\lambda|=|\mu|=|\pi| \leq m^{3}$, and

(vi) $\lambda$ is not a hook.

Furthermore:

THEOREM 1.8. Assuming coNP $\neq N P$, the set of partition triples satisfying constraints Theorem 1.7(i) (vi) as well as

(vii) $\left(\lambda^{T}, \mu^{T}, \mu\right) \in \operatorname{Kron}\left(m^{\prime}\right)$, where $m^{\prime}$ is the maximum of the heights of $\lambda^{T}, \mu^{T}$ and $\mu$, and

(viii) $\left(\lambda, \mu^{T}, \mu^{T}\right) \in \operatorname{Kron}\left(m^{\prime \prime}\right)$, where $m^{\prime \prime}$ is the maximum of the heights of $\lambda$ and $\mu^{T}$,

is superpolynomial in $m$, as $m \rightarrow \infty$.

The constraints (iv), (vii), and (viii) together guarantee that the vanishing of $k_{\mu, \mu}^{\lambda}$ cannot be directly shown using the defining inequalities of the Kronecker cone (Berenstein \& Sjamaar 2000, Klyachko 2004; Ressayre 2010; Vergne \& Walter 2017) in conjunction with the known symmetries (1.1) and (1.2).

Our proof of Theorem 1.7 shows that the partition triples $(\lambda, \mu, \mu)$ satisfying the constraints therein can even be constructed explicitly. This means there is a one-to-one map from the set of Boolean strings of length $\leq m^{a}$ to the set of partitions triples 
$(\lambda, \mu, \mu)$ with properties (i) (vi) that can be computed in poly $(m)$ time.

While Theorem 1.7 shows existence of superpolynomially many partition triples satisfying the constraints therein, the density of such partition triples is exponentially small, since $a$ therein is much smaller than 1 (see Example 5.4). This may explain why vanishing Kronecker coefficients with the partition triples in the Kronecker cone occur so rarely in computer experiments, as observed in Ikenmeyer (2012).

1.6. Proof technique. Theorem 1.3 is proved by extending the NP-completeness technique in Brunetti et al. (2001) in conjunction with the fundamental lower and upper bounds on Kronecker coefficients established in Bürgisser \& Ikenmeyer (2013); Manivel (1997); Vallejo (2000). Theorem 1.5 is a byproduct of this proof.

A refined form of Theorem 1.3 lies at the heart of the proof of Theorem 1.7. Specifically, we show in Theorem 4.2 that the problem of deciding positivity of $k_{\mu, \pi}^{\lambda}$ remains NP-hard under polynomialtime many-one reductions (Karp 1972) even when the partitions $(\lambda, \mu, \pi)$ are required to satisfy the constraints Theorem 1.7(ii) (vi). This is done by extending the proof technique of Theorem 1.7 using the result in Bürgisser et al. (2011a) that $(\lambda, \delta(\lambda), \delta(\lambda))$, for $|\lambda|$ divisible by $r$, lies in the Kronecker cone whenever the height of $\lambda$ is $\leq r^{2}$. By Fortune (1979), if there exists a co-sparse NP-complete language under polynomial-time many-one-reductions, then $\mathrm{P}=\mathrm{NP}$. (Here we call a language sparse if the number of strings in it of bitlength $\leq N$ is bounded by a fixed polynomial in $N$. It is called co-sparse if its complement is sparse.) Hence Theorem 4.2 in conjunction with Fortune (1979) implies that the set of partition triples satisfying the constraints Theorem 1.7(i) (vi) is non-sparse, i.e., has size superpolynomial in $m$, assuming that $\mathrm{P} \neq \mathrm{NP}$.

To prove Theorem 1.7, we have to discard of the assumption that $\mathrm{P}=\mathrm{NP}$ and replace the superpolynomial bound by $\Omega\left(2^{m^{a}}\right)$ bound for some $a>0$. This is done in Theorem 5.2 by exhibiting a polynomial-time one-one reduction from the 3D MATCHING problem (Garey \& Johnson 1979) to the problem of deciding positivity of Kronecker coefficients, with the partition triples satisfying the constraints Theorem 1.7(ii) (vi), where a polynomial-time one-one 
reduction means an injective polynomial-time many-one reduction. Hence, the $\Omega\left(2^{m^{a}}\right)$ bound in Theorem 1.7 follows from a similar lower bound on the number of instances of the 3D MATCHING problem with a "NO" answer. The proof automatically shows that $\Omega\left(2^{m^{a}}\right)$ partition triples satisfying the constraints in Theorem 1.7 can be constructed explicitly. This follows by fixing a suitable set of $2^{N^{b}}$ instances, for some constant $b>0$, of the 3D MATCHING problem of bitlength $\leq N$ with "NO" answer, and mapping them injectively, via a sequence of polynomial time one-one reductions, to $\Omega\left(2^{m^{a}}\right)$ such partition triples.

Theorem 1.8 is proved by extending the proof of Theorem 1.7 using an auxiliary result, Lemma 5.5, which extends the hardness vs. non-sparseness result in Fortune (1979), together with the result in Bürgisser et al. (2017) which asserts that the membership problem for the Kronecker cone is in NP $\cap$ coNP.

\subsection{Effectiveness of the explicit proof strategy. Perhaps} the most novel aspect of this paper is the synthesis of the representation theory of Kronecker coefficients with the theory of NP-completeness to prove unconditionally existence of superpolynomially many partition triples in the Kronecker cone with vanishing Kronecker coefficients.

In principle, the existence of partition triples satisfying the constraints in Theorem 1.7 may be proved by a nonconstructive technique. Yet, the only way we can prove this existence at present is by constructing such partitions explicitly, using the theory of algorithms, as done in the proof of Theorem 1.7. Thus this proof illustrates effectiveness of the explicit proof strategy of GCT $\mathrm{Mul}$ muley 2010a b, 2011) in a nontrivial setting.

1.8. Organization. The rest of this article is organized as follows. Section 2 describes the lower and upper bounds for the Kronecker coefficients that are needed for the proofs of Theorem 1.3 and Theorem 1.5. These proofs are given in Section 3. A refinement of Theorem 1.3, which is needed for the proof of Theorem 1.7, is proved in Section 4, Theorem 1.7 and Theorem 1.8 are proved in Section 5. Section 6 proves additional results in support of the conjecture in Mulmuley (2010b) that the problem of deciding 
positivity of rectangular Kronecker coefficients is in $P$.

\section{Lower and upper bounds for the Kronecker coefficient}

In this section, we give representation-theoretic proofs of some known lower and upper bounds from Bürgisser \& Ikenmeyer (2013); Manivel (1997); Vallejo (2000) for the Kronecker coefficients. These bounds as well as their representation-theoretic interpretation given here will play a crucial role in the proofs of Theorem 1.3 and Theorem 1.5 in Section 3. We begin with the following well-known result (whose proof we include for the sake of completeness):

Lemma 2.1. Let $\lambda, \mu, \pi$ denote Young diagrams, each with $n$ boxes and no more than $r$ columns. Then the Kronecker coefficient $k_{\mu, \pi}^{\lambda}$ is equal to the multiplicity of the irreducible representation $V_{\lambda^{T}}(\mathrm{GL}(r)) \otimes$ $V_{\mu^{T}}(\mathrm{GL}(r)) \otimes V_{\pi^{T}}(\mathrm{GL}(r))$ of $\mathrm{GL}(r)^{3}$ in the anti-symmetric subspace $\bigwedge^{n}\left(\mathbb{C}^{r}\right)^{\otimes 3}$.

Proof. Let $\tilde{k}_{\alpha, \beta, \gamma}^{\kappa}$ denote the multiplicity of the irreducible representation $V_{\alpha}(\mathrm{GL}(r)) \otimes V_{\beta}(\mathrm{GL}(r)) \otimes V_{\gamma}(\mathrm{GL}(r))$ of $\mathrm{GL}(r)^{3}$ in the Weyl module $V_{\kappa}\left(\mathrm{GL}\left(r^{3}\right)\right)$. Our original definition of the Kronecker coefficient is easily seen to be equivalent to $\tilde{k}_{\lambda, \mu, \pi}^{(n)}$ (e.g., Walter (2014), but this is standard), and so we need to show that

$$
\tilde{k}_{\lambda, \mu, \pi}^{(n)}=\tilde{k}_{\lambda^{T}, \mu^{T}, \pi^{T}}^{\left(1^{n}\right)}
$$

Given a partition $\kappa$, let $[\kappa]$ denote the Specht module (i.e., an irreducible representation) of $S_{n}$. By Schur-Weyl duality, $\tilde{k}_{\alpha, \beta, \gamma}^{\kappa}$ is also equal to the multiplicity of $[\kappa]$ in the triple tensor product $[\alpha] \otimes[\beta] \otimes[\gamma]$. Since the representations of the symmetric group are self-dual, this shows that:

$$
\tilde{k}_{\alpha, \beta, \gamma}^{\kappa}=\operatorname{dim}([\alpha] \otimes[\beta] \otimes[\gamma] \otimes[\kappa])^{S_{n}}
$$

Since $\left[\lambda^{T}\right]=[\lambda] \otimes\left[\left(1^{n}\right)\right],\left[\left(1^{n}\right)\right] \otimes\left[\left(1^{n}\right)\right]=[(n)]$, and $[(n)]$ is the trivial representation, $(2.2)$ follows at once. 
Given a (finite) point set $P \subseteq\{0, \ldots, r-1\}^{3}$, let $x_{P}(i), 0 \leq$ $i \leq r-1$, be the number of points in $P$ with the $x$-coordinate $i$. We call $x_{P}=\left(x_{P}(0), \ldots, x_{P}(r-1)\right)$ the $x$-marginal of $P$. We similarly define the $y$-marginal $y_{P}$ and the $z$-marginal $z_{P}$. The triple $\left(x_{P}, y_{P}, z_{P}\right)$ is called the marginals of $P$.

Definition 2.3. We define $t_{\mu, \pi}^{\lambda}$ as the number of point sets $P \subseteq$ $\{0, \ldots, r-1\}^{3}$ with marginals $\left(\lambda^{T}, \mu^{T}, \pi^{T}\right)$.

Note that $\lambda_{i}^{T}$ is the number of boxes in the $i$-th column of $\lambda$.

The coefficients $t_{\mu, \pi}^{\lambda}$ have a pleasant representation-theoretical interpretation that is closely related to Lemma 2.1. To see this, observe that we can associate with any point set

$$
P=\left\{\left(x_{1}, y_{1}, z_{1}\right), \ldots,\left(x_{n}, y_{n}, z_{n}\right)\right\} \subseteq\{0, \ldots, r-1\}^{3}
$$

of cardinality $n$ the following vector:

$$
\psi_{P}=\bigwedge_{j=1}^{n} e_{x_{j}} \otimes e_{y_{j}} \otimes e_{z_{j}} \in \bigwedge^{n}\left(\mathbb{C}^{r}\right)^{\otimes 3},
$$

where $\left\{e_{i}\right\}$ is the standard basis of $\mathbb{C}^{r}$. The vectors $\psi_{P}$ form a basis of $\bigwedge^{n}\left(\mathbb{C}^{r}\right)^{\otimes 3}$ as $P$ ranges over all such point sets. Moreover, each $\psi_{P}$ is a weight vector for the $G L(r)^{3}$-action, whose weight is given by the marginals of the point set $P$ (see Section 1.1 for the definition of a weight vector). Thus we obtain the following result.

Lemma 2.4. Let $\lambda, \mu$, and $\pi$ denote Young diagrams with $n$ boxes each and no more than $r$ columns. Then $t_{\mu, \pi}^{\lambda}$ is equal to the weight multiplicity of $\left(\lambda^{T}, \mu^{T}, \pi^{T}\right)$ in the anti-symmetric $G L(r)^{3}$-module $\bigwedge^{n}\left(\mathbb{C}^{r}\right)^{\otimes 3}$.

Following Vallejo (2000), we call a subset $P \subseteq\{0, \ldots, r-1\}^{3}$ a pyramid if, for any $(x, y, z) \in P$ and $0 \leq x^{\prime} \leq x, 0 \leq y^{\prime} \leq y$, $0 \leq z^{\prime} \leq z$, we have that $\left(x^{\prime}, y^{\prime}, z^{\prime}\right) \in P$. (It would also be natural to call such $P$ a 3-partition; cf. Manivel (1997).)

DEFINITION 2.5. Let $p_{\mu, \pi}^{\lambda}$ denote the number of pyramids with marginals $\left(\lambda^{T}, \mu^{T}, \pi^{T}\right)$. 
From our representation-theoretic interpretation, we directly obtain the following fundamental bounds, which were proved previously using different methods in Bürgisser \& Ikenmeyer (2013); Manivel (1997) (cf. Vallejo 2000):

LEMma 2.6. For all partitions $\lambda, \mu, \pi$, we have $p_{\mu, \pi}^{\lambda} \leq k_{\mu, \pi}^{\lambda} \leq t_{\mu, \pi}^{\lambda}$.

ProOF. The upper bound on $k_{\mu, \pi}^{\lambda}$ follows directly from Lemma 2.1 and Lemma 2.4, since the irreducible representations of $G L(r)^{3}$ in $\bigwedge^{n}\left(\mathbb{C}^{r}\right)^{\otimes 3}$ are in one-to-one correspondence with their highest weight vectors, and every highest weight vector is a weight vector.

For the lower bound, suppose that

$$
P=\left\{\left(x_{1}, y_{1}, z_{1}\right), \ldots,\left(x_{n}, y_{n}, z_{n}\right)\right\} \subseteq\{0, \ldots, r-1\}^{3}
$$

is a pyramid with marginals $\left(\lambda^{T}, \mu^{T}, \pi^{T}\right)$. We will show that $\psi_{P}$ is not only a weight vector, but in fact a highest weight vector. For this, we need to argue that $\psi$ is annihilated by all raising operators (cf. Section 1.1). Thus consider $\left(E_{x^{\prime}, x}, 0,0\right)$, where $E_{x^{\prime}, x}$ denotes the upper triangular matrix with a single 1 in the $x^{\prime}$-th row and $x$-th column, and otherwise zero (here $x^{\prime}<x$ ). Its action on $\psi_{P}$ is given by

$$
\begin{aligned}
& \left(E_{x^{\prime}, x}, 0,0\right) \cdot \psi_{P} \\
= & \sum_{j=1}^{n}(-1)^{j-1} E_{x^{\prime}, x} e_{x_{j}} \otimes e_{y_{j}} \otimes e_{z_{j}} \wedge \bigwedge_{j^{\prime}=1, j^{\prime} \neq j}^{n} e_{x_{j^{\prime}}} \otimes e_{y_{j^{\prime}}} \otimes e_{z_{j^{\prime}}} \\
= & \sum_{j=1}^{n}(-1)^{j-1} \delta_{x, x_{j}} e_{x^{\prime}} \otimes e_{y_{j}} \otimes e_{z_{j}} \wedge \bigwedge_{j^{\prime}=1, j^{\prime} \neq j}^{n} e_{x_{j^{\prime}}} \otimes e_{y_{j^{\prime}}} \otimes e_{z_{j^{\prime}}}=0,
\end{aligned}
$$

since each summand vanishes individually. Indeed, if $x \neq x_{j}$ then $\delta_{x, x_{j}}=0$ and so the summand is zero. Otherwise, if $x=x_{j}$ then $\left(x_{j}, y_{j}, z_{j}\right) \in P$ and $x^{\prime}<x$ imply that $\left(x^{\prime}, y_{j}, z_{j}\right) \in P$ by the pyramid condition; therefore $e_{x^{\prime}} \otimes e_{y_{j}} \otimes e_{z_{j}}$ appears twice in the wedge product and so the summand vanishes as well. The same argument applies to the other generators $\left(0, E_{y^{\prime}, y}, 0\right)$ and $\left(0,0, E_{z^{\prime}, z}\right)$ of $\mathfrak{n}$. Thus we conclude that the pyramid condition ensures that $\psi_{P}$ is a highest weight vector. 
Corollary 2.7. Let $\lambda, \mu, \pi$ be partitions such that any point set with marginals $\left(\lambda^{T}, \mu^{T}, \pi^{T}\right)$ is necessarily a pyramid. Then $k_{\mu, \pi}^{\lambda}=t_{\mu, \pi}^{\lambda}=p_{\mu, \pi}^{\lambda}$.

\section{Kronecker coefficients with \#P-formulae}

In this section, we prove Theorem 1.3 and Theorem 1.5.

For this, we first derive a sufficient condition on the marginals $\left(\lambda^{T}, \mu^{T}, \pi^{T}\right)$ such that any compatible point set is necessarily a pyramid (and hence Corollary 2.7 is applicable). Adapting the approach of Brunetti et al. (2001), we consider a point set $P$ such that $P_{r} \subseteq P \subsetneq P_{r+1}$, where

$$
P_{r}=\left\{(x, y, z) \in\{0, \ldots, r-1\}^{3}: x+y+z \leq r-1\right\}
$$

denotes the simplex of side length $r \geq 1$. Let $n$ denote the total number of points in $P$. Then the projection of the barycenter $b_{P}:=\sum_{p \in P} p$ of $P$ onto the diagonal $(1,1,1)$ can be computed as follows:

$$
b_{P} \cdot(1,1,1)=b_{r} \cdot(1,1,1)+r\left(n-\left|P_{r}\right|\right)=: p(n),
$$

where $b_{r}$ denotes the barycenter of the simplex $P_{r}$. Note that this formula depends only $n$, the number of points in the point set $P$. We can thus define a function $p(n)$ by (3.1), first for all $n$ such that $\left|P_{r}\right| \leq n<\left|P_{r+1}\right|$, and then, by varying $r$, for all $n$. Explicitly,

$$
p(n)=b_{r(n)} \cdot(1,1,1)+r(n)\left(n-\left|P_{r(n)}\right|\right),
$$

where $r(n)$ is the maximal $r$ such that $\left|P_{r}\right|=r(r+1)(r+2) / 6 \leq n$.

Definition 3.3. Let us call $(\lambda, \mu, \pi)$, with $|\lambda|=|\mu|=|\pi|=n \neq 0$, simplex-like if there exists some $r$ such that the Young diagrams of $\lambda, \mu$, and $\pi$ have at most $r+1$ columns, and

$$
\sum_{i=0}^{r} i \lambda_{i}^{T}+\sum_{j=0}^{r} j \mu_{j}^{T}+\sum_{k=0}^{r} k \pi_{k}^{T}=p(n) .
$$

Whether $(\lambda, \mu, \pi)$ is simplex-like can be checked in polynomial time (even assuming that $\lambda, \mu$ and $\pi$ are given in binary).

The following lemma justifies the term "simplex-like". 
Lemma 3.4. Let $(\lambda, \mu, \pi)$ be simplex-like. Then any point set $P$ with marginals $\left(\lambda^{T}, \mu^{T}, \pi^{T}\right)$ is necessarily of the form $P_{r} \subseteq P \subsetneq$ $P_{r+1}$, for some $r \geq 1$. In particular, $P$ is a pyramid.

Proof. The level sets of the function $p \mapsto p \cdot(1,1,1)$ restricted to the positive octant are precisely the faces $\left\{(x, y, z) \in \mathbb{N}^{3}: x+y+z=\right.$ $k-1\}$ of the simplices $P_{k}$. Thus it is geometrically obvious that for an arbitrary point set $P$ with $n$ elements, $b_{P} \cdot(1,1,1)$ is never smaller than $p(n)$, and that it attains this minimum if and only if $P_{r} \subseteq P \subsetneq P_{r+1}$, for some $r \geq 1$ (cf. Brunetti et al. 2001). Therefore, it suffices to show that our assumptions imply that $b_{P} \cdot(1,1,1)=p(n)$. This is indeed true as shown in the following computation, which relies on the fact that the barycenter is purely a function of the marginals:

$$
b_{P} \cdot(1,1,1)=\sum_{(x, y, z) \in P}(x+y+z)=\sum_{i=0}^{r} i \lambda_{i}^{T}+\sum_{j=0}^{r} j \mu_{j}^{T}+\sum_{k=0}^{r} k \pi_{k}^{T}=p(n) .
$$

The last step follows because $(\lambda, \mu, \pi)$ is simplex-like.

Theorem 3.5. Let $(\lambda, \mu, \pi)$ be simplex-like. Then $k_{\mu, \pi}^{\lambda}=t_{\mu, \pi}^{\lambda}=$ $p_{\mu, \pi}^{\lambda}$. In particular, this family of Kronecker coefficients has a $\# P$-formula. Here, $\lambda, \mu$ and $\pi$ can be given in unary or binary.

Proof. This follows from Lemma 3.4, Corollary 2.7, and the fact that $t_{\mu, \pi}^{\lambda}$ has a \#P-formula. The last assertion follows because the bit-length of the unary specification of a simplex-like partition triple is polynomial in the bit-length of its binary specification.

One important class of simplex-like marginals is the following. Let $\left(\varphi^{T}, \varphi^{T}, \varphi^{T}\right)$ denote the marginals of the simplex $P_{2 r}$, where $r \geq 1$. Define

$$
\lambda^{T}:=\varphi^{T}+\left(d_{2 r}, \ldots, d_{0}\right), \quad \mu^{T}=\pi^{T}:=\varphi^{T}+\left(1^{r+1}, 0^{r}\right),
$$

where $d=\left(d_{0}, \ldots, d_{2 r}\right) \in \mathbb{N}^{2 r+1}$ is such that $\sum_{k} d_{k}=r+1$ and $\sum_{k} k d_{k}=r(r+1)$-this also implies that $\sum_{k} k d_{2 r-k}=r(r+1)$. It is not hard to see that these marginals are simplex-like. Indeed,

$$
\sum_{i=0}^{2 r} i \lambda_{i}^{T}+\sum_{j=0}^{2 r} j \mu_{j}^{T}+\sum_{k=0}^{2 r} k \pi_{k}^{T}
$$




$$
\begin{aligned}
& =b_{2 r} \cdot(1,1,1)+\sum_{i=0}^{2 r} i d_{2 r-i}+\sum_{j=0}^{r} j+\sum_{k=0}^{r} k \\
& =b_{2 r} \cdot(1,1,1)+2 r(r+1) .
\end{aligned}
$$

Since $\left|P_{2 r}\right| \leq n=\left|P_{2 r}\right|+(r+1)<\left|P_{2 r+1}\right|$, this is indeed equal to $p(n)$, where $n$ is the number of boxes of each of $\lambda, \mu$, and $\pi$ (compare with (3.2) . These marginals arise when embedding permutation matrices on top of the simplex $P_{2 r}$, and in Brunetti et al. (2001) it was shown using this construction that:

TheOrem 3.7 (Brunetti et al. $\mid$ 2001). The problem of deciding positivity of $t_{\mu, \pi}^{\lambda}$, given $\lambda, \mu, \pi$ in unary, is NP-hard with respect to polynomial-time many-one reductions, even when $\left(\lambda^{T}, \mu^{T}, \pi^{T}\right)$ is restricted to be of the form (3.6).

Thus it follows at once from Theorem 3.5, in conjunction with this result, that:

THEOREM 3.8. The problem of deciding positivity of the Kronecker coefficient $k_{\mu, \pi}^{\lambda}$, given $\lambda, \mu, \pi$ in unary, is NP-hard with respect to polynomial-time many-one reductions, even when $\left(\lambda^{T}, \mu^{T}, \pi^{T}\right)$ is restricted to be of the form (3.6).

This proves Theorem 1.3. Theorem 1.5 follows from this result and Theorem 3.5 .

\section{Refined NP-hardness result}

Let RESTRICTED KRONECKER be the problem of deciding positivity of $k_{\mu, \pi}^{\lambda}$, when $\lambda, \mu, \pi$ satisfy the constraints Theorem 1.7(ii) (vi). Specifically:

Definition 4.1. Fix a constant $0<\epsilon \leq 1$. Then Restricted KRONECKER is the problem of deciding, given $m \in \mathbb{N}$ and partitions $\lambda, \mu, \pi$ in unary, with $\mathrm{ht}(\mu)=\mathrm{ht}(\pi)=m$, whether the Kronecker coefficient $k_{\mu, \pi}^{\lambda}$ is positive, assuming that:

(ii) $\mu=\pi$,

(iii) $\mathrm{ht}(\lambda) \leq m^{\epsilon}$, 
(iv) $(\lambda, \mu, \pi)$ is in the Kronecker cone $\operatorname{Kron}(m)$,

(v) $|\lambda|=|\mu|=|\pi| \leq m^{3}$, and

(vi) $\lambda$ is not a hook.

For the proof of Theorem 1.7, we need the following refinement of Theorem 3.8:

TheOrem 4.2. Restricted KroneCKer is NP-hard with respect to polynomial-time many-one reductions.

We first we need some auxiliary results.

LEMmA 4.3. Let $\lambda$ and $\mu$ be partitions so that $\mathrm{ht}(\lambda) \leq h^{2}$, where $h$ is the height of the smallest column of $\mu$. Then $(\lambda, \mu, \mu)$ is in the Kronecker cone $\operatorname{Kron}(l)$, where $l=\max \{\operatorname{ht}(\lambda), \operatorname{ht}(\mu)\}$.

ProOF. It is shown in Bürgisser et al. (2011a) that $(\lambda, \delta, \delta)$ is in the Kronecker cone whenever $\delta$ is a rectangle of height at least $h$, and $h t(\lambda) \leq h^{2}$. As the Kronecker cone is a cone, this is also true if we rescale each of $\lambda$ and $\delta$ by an arbitrary positive number.

Let us write $\mu$ as a sum of rectangles $\delta^{(1)}+\cdots+\delta^{(k)}$, where our assumption implies that each $\delta^{(j)}$ has height at least $h$. It is easy to see that $\lambda$ can be written as a sum $\lambda^{(1)}+\cdots+\lambda^{(k)}$, where each $\lambda^{(j)}$ is a rational partition with the same size as $\delta^{(j)}$ (i.e., $\left|\lambda^{(j)}\right|=\left|\delta^{(j)}\right|$ ), and with no more than $h^{2}$ rows. By the preceding argument, each $\left(\lambda^{(j)}, \delta^{(j)}, \delta^{(j)}\right)$ is in the Kronecker cone $\operatorname{Kron}(l)$. As cones are closed under addition, $(\lambda, \mu, \mu)$ is likewise in the Kronecker cone.

Next, we generalize Theorem 3.5 to a larger class of marginals. Let $(\lambda, \mu, \pi)$ be simplex-like, and let $P$ be a corresponding point set with marginals $\left(\lambda^{T}, \mu^{T}, \pi^{T}\right)$, so that $P_{r} \subseteq P \subsetneq P_{r+1}$ for some $r$ (Lemma 3.4). Let $Q$ denote the following point set obtained by adjoining $P$ to a rectangular box of size $a \times b \times c$, where $b, c \geq r+1$ :

$$
\begin{aligned}
& Q=\{0, \ldots, a-1\} \times\{0, \ldots, b-1\} \times\{0, \ldots, c-1\} \\
& \cup\{(a+x, y, z):(x, y, z) \in P\} .
\end{aligned}
$$

Then the marginals of $Q$ are given by $\left(\tilde{\lambda}^{T}, \tilde{\mu}^{T}, \tilde{\pi}^{T}\right)$, where

$$
\tilde{\lambda}^{T}=\left((b c)^{a}, \lambda^{T}\right), \quad \tilde{\mu}^{T}=\mu^{T}+\left((a c)^{b}\right), \quad \tilde{\pi}^{T}=\pi^{T}+\left((a b)^{c}\right),
$$


so that

$$
\tilde{\lambda}=\left(a^{b c}\right)+\lambda, \quad \tilde{\mu}=\left(b^{a c}, \mu\right), \quad \tilde{\pi}=\left(c^{a b}, \pi\right) .
$$

Definition 4.6. We call $(\tilde{\lambda}, \tilde{\mu}, \tilde{\pi})$ pedestalled-simplex-like if it is of the form (4.5) for some simplex-like $(\lambda, \mu, \pi)$ with at most $r+1$ columns each, $a \geq 0$, and $b, c \geq r+1$.

Then we have the following generalization of Lemma 3.4.

Lemma 4.7. Let $(\tilde{\lambda}, \tilde{\mu}, \tilde{\pi})$ be pedestalled-simplex-like, i.e., of the form (4.5) for some simplex-like $(\lambda, \mu, \pi)$. Then (4.4) defines a bijection between point sets $P$ with marginals $\left(\lambda^{T}, \mu^{T}, \pi^{T}\right)$ and point sets $Q$ with marginals $\left(\tilde{\lambda}^{T}, \tilde{\mu}^{T}, \tilde{\pi}^{T}\right)$. In particular, any such point set $Q$ is a pyramid.

ProOF. It suffices to show that any point set $Q$ with marginals $(\tilde{\lambda}, \tilde{\mu}, \tilde{\pi})$ is of the form (4.4). For this, observe that according to the definition of $\tilde{\lambda}$, the first $a$ x-slices of $Q$ contain exactly $b c$ points each. On the other hand, the definition of $\tilde{\mu}$ and $\tilde{\pi}$ implies that there are at most $b$ non-zero y-slices and at most $c$ non-zero z-slices, so that $Q$ is a point set in $\mathbb{N} \times\{0, \ldots, b-1\} \times\{0, \ldots, c-1\}$. It follows that the first $a$ x-slices must each be filled completely without holes by rectangles of size $b \times c$. Therefore we may write $Q$ in the form (4.4), and it is clear that $P$ has the correct marginals $\left(\lambda^{T}, \mu^{T}, \pi^{T}\right)$. Since $(\lambda, \mu, \pi)$ is simplex-like, $P_{r} \subseteq P \subseteq P_{r+1}$ Lemma 3.4). Finally, observe that any $Q$ of the form (4.4) is clearly a pyramid.

The following result generalizes Theorem 3.5.

THEOREM 4.8. Let $(\tilde{\lambda}, \tilde{\mu}, \tilde{\pi})$ be pedestalled-simplex-like, i.e., of the form (4.5) for some simplex-like $(\lambda, \mu, \pi)$. Then:

$$
k_{\tilde{\mu}, \tilde{\pi}}^{\tilde{\lambda}}=t_{\tilde{\mu}, \tilde{\pi}}^{\tilde{\lambda}}=t_{\mu, \pi}^{\lambda}=k_{\mu, \pi}^{\lambda}
$$

ProOF. The first equality follows from Corollary 2.7, as according to Lemma 4.7 any point set with marginals $\left(\tilde{\lambda}^{T}, \tilde{\mu}^{T}, \tilde{\pi}^{T}\right)$ is necessarily a pyramid. The middle equality follows from Lemma 4.7. The last equality is Theorem 3.5. 
We can now give the proof of Theorem 4.2.

Proof Theorem 4.2). We apply the pedestal construction to marginals of the form (3.6). Let us choose a rectangular box of size $c \times s \times s$, where $s=2 r+1$. That is, we set

$$
\begin{aligned}
\lambda^{T} & =\left(\left(s^{2}\right)^{c},\left(\varphi^{T}+\left(d_{2 r}, \ldots, d_{0}\right)\right)\right), \\
\mu^{T}=\pi^{T} & =\left((c s)^{s}\right)+\varphi^{T}+\left(1^{r+1}, 0^{r}\right),
\end{aligned}
$$

for some $d=\left(d_{0}, \ldots, d_{2 r}\right) \in \mathbb{N}^{2 r+1}$ such that $\sum_{k} d_{k}=r+1$ and $\sum_{k} k d_{k}=r(r+1)$, where $\varphi^{T}$ denotes the marginal of the simplex $P_{2 r}=P_{s-1}$. Furthermore, we set $c:=\left\lceil s^{2 / \epsilon-1}\right\rceil$.

The problem of deciding positivity of $k_{\mu, \pi}^{\lambda}$, with $(\lambda, \mu, \pi)$ restricted as above, is NP-hard, since by Theorem 4.8, these Kronecker coefficients agree with the ones in Theorem 3.8, and we can transform instances of the latter to instances of the former in polynomial time (as $\epsilon$ is fixed).

We now verify that the five constraints in Definition 4.1 are all satisfied. The first is clearly satisfied. For the second,

$$
\mathrm{ht}(\lambda)=s^{2} \leq(c s)^{\epsilon} \leq \mathrm{ht}(\mu)^{\epsilon}=m^{\epsilon},
$$

by our choice of $c=c(s)$. The third follows from Lemma 4.3, as ht $(\lambda)=s^{2}$, while every column in $\mu$ is of height at least $c s \geq s$. The fourth follows, since

$$
\begin{aligned}
& |\lambda|=c s^{2}+\left|\varphi^{T}\right|+\sum_{k} d_{k}=c s^{2}+\frac{(s-1) s(s+1)}{6}+r+1 \\
& \leq(c s)^{3} \leq \operatorname{ht}(\mu)^{3}=m^{3},
\end{aligned}
$$

assuming that $r$ is large enough. Finally, it is clear that $\lambda$ is not a hook.

\section{Construction of vanishing Kronecker coefficients with partition triples in the Kronecker cone}

In this section, we prove Theorem 1.7 and Theorem 1.8. 
5.1. Proof of Theorem 1.7. By Fortune (1979), if there exists a co-sparse NP-complete language under polynomial-time manyone-reductions, then $\mathrm{P}=\mathrm{NP}$. Theorem 4.2 , in conjunction with this result, implies that, assuming $\mathrm{P} \neq \mathrm{NP}$, the set of partitions triples satisfying the constraints Theorem 1.7(i) (vi) is non-sparse, i.e, its cardinality is superpolynomial in $m$. (The result in Fortune 1979) applies to NP-complete sets, rather than NP-hard sets. But we can still apply this result to the NP-complete set of simplex-like partition triples (cf. Theorem 3.8) with positive Kronecker coefficients to get the desired conclusion.) To prove Theorem 1.7, we have to get rid of the assumption that $\mathrm{P} \neq \mathrm{NP}$ and replace the superpolynomial bound by $\Omega\left(2^{m^{a}}\right)$ bound, for some positive constant $a$. This will be achieved by Lemma 5.1 and Theorem 5.2 below.

Recall from Garey \& Johnson (1979) that the 3D Matching problem is to decide, given a set $M \subseteq W \times X \times Y$, where $W$, $X, Y$ are disjoint sets of size $q$, whether $M$ contains a (perfect) matching, i.e., a subset $M^{\prime} \subseteq M$ of size $q$ such that no two elements of $M^{\prime}$ agree in any coordinate. Without loss of generality, we assume henceforth that each element in $W \cup X \cup Y$ appears in some triple of $M$. We denote instances of 3D MATCHING by tuples $(M, W, X, Y, q)$. It is known that the 3D MATCHING problem is NP-complete (Garey \& Johnson 1979).

LEMma 5.1. The number of instances $(M, W, X, Y, q)$ of the 3D MATCHING problem with total bit-length $\leq N$ such that $M$ does not have a matching is $\Omega\left(2^{N^{b}}\right)$ for some positive constant $b<1$.

Furthermore, such instances can be constructed explicitly. That is, for some positive constant $b<1$, there is a polynomial-timecomputable one-to-one function that maps any pair of the form $(N, \sigma)$, where $N$ is a positive integer and $\sigma$ is a binary string of length $\leq N^{b}$, to an instance of 3D MATCHING problem without matching of bitlength $\leq N$.

Proof. Consider any fixed instance $\left(M_{0}, W_{0}, X_{0}, Y_{0}, q_{0}\right)$ of $3 \mathrm{D}$ MATCHING, such that $M_{0}$ does not have a matching. Its bitlength is thus a constant. Given any instance $(M, W, X, Y, q)$ of 3D MATCHING, with $W, X$ and $Y$ disjoint from $W_{0}, X_{0}$ and $Y_{0}$, consider the padded instance $\left(M \cup M_{0}, W \cup W_{0}, X \cup X_{0}, Y \cup Y_{0}, q+q_{0}\right)$. Clearly, 


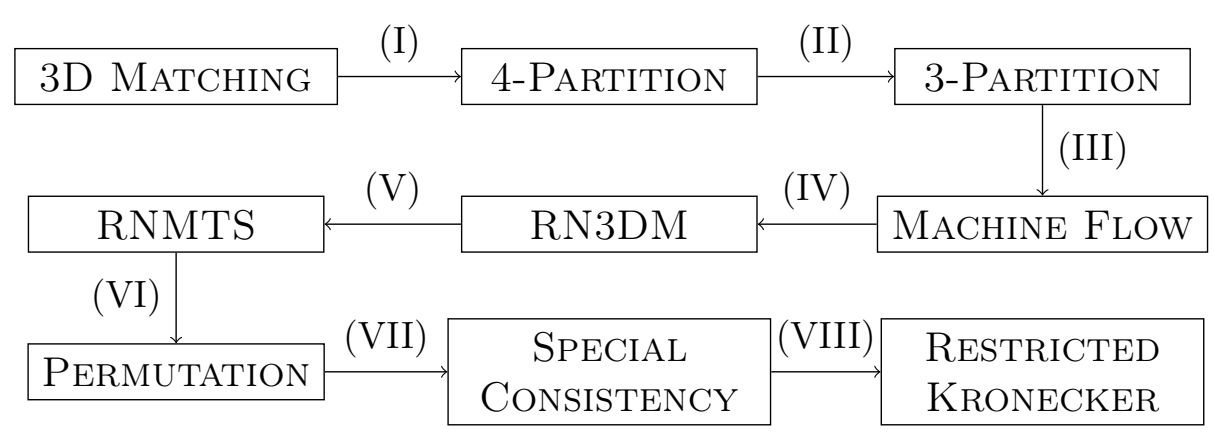

Figure 5.1: Sequence of reductions used in the proof of Theorem 5.2.

$M \cup M_{0}$ also does not have a matching. The number of instances of the form $\left(M \cup M_{0}, W \cup W_{0}, X \cup X_{0}, Y \cup Y_{0}, q+q_{0}\right)$ with bitlength $\leq N$ is clearly $\Omega\left(2^{N^{b}}\right)$ for some positive constant $b<1$. This is because, for a given $q$, the total number of instances of the form $(M, W, X, Y, q)$ is $2^{q^{3}}$, and the bit-length of the specification of any instance of this form is $O\left(q^{3}\right)$. (We assume that $M$ is specified by its $q \times q \times q$ adjacency matrix.) Furthermore, it is easy to show that the padded instances of the form $\left(M \cup M_{0}, W \cup W_{0}, X \cup X_{0}, Y \cup Y_{0}, q+q_{0}\right)$ can be constructed explicitly.

Recall (cf. Section 1.6) that a polynomial-time one-one reduction means an injective polynomial-time many-one reduction.

THEOREM 5.2. There exists a polynomial-time one-one reduction $\phi$ from the set of instances $(M, W, X, Y, q)$ of 3D MATCHING of total bit-length $n$ to the set of partition triples $(\lambda, \mu, \pi)$ satisfying the conditions Theorem 1.7(ii) (vi) (i.e., instances of RESTRICTED KRONECKER), with $m=\operatorname{poly}(n)$, such that $M$ contains a matching iff the Kronecker coefficient associated with the partition triple $\phi(E)$ is positive.

Proof. Since 3D Matching is in NP, it follows from Theorem 4.2 that there exists a polynomial-time many-one reduction $\phi$ from 3D MATCHING to the RESTRICTED KRONECKER problem of deciding positivity of the Kronecker coefficient $k_{\mu, \pi}^{\lambda}$, with $(\lambda, \mu, \pi)$ satisfying the constraints Theorem 1.7(ii) (vi), We have to show that this reduction $\phi$ can be chosen to be injective. We can obtain 
such an injective reduction $\phi$ by composing the following sequence of polynomial-time computable one-one-reductions (Figure 5.1):

(I) From 3D Matching to 4-PARtition (cf. Theorem 4.3 in Garey \& Johnson 1979):

The 4-PARTItion problem is to decide, given a set $A$ of size $4 m$, a positive integer bound $B$, a positive integer size $s(a)$ for each $a \in A$ such that $B / 5<s(a)<B / 3$ and $\sum_{a \in A} s(a)=$ $m B$, whether $A$ can be partitioned into $m$ disjoint subsets $A_{1}, \ldots, A_{m}$, each of size four, such that, for each $1 \leq i \leq m$, $\sum_{a \in A_{i}} s(a)=B$. We denote such an instance of 4-PARTition by the tuple $(A, m, B, s)$.

The reduction in Garey \& Johnson (1979) maps a given instance $(M, W, X, Y, q)$ of $3 \mathrm{D}$ MATCHING to an instance $(A, m, B, s)$ of 4-partition, where:

- The set $A$ has $4|M|=O\left(q^{3}\right)$ elements, one for each occurrence of a member of $W \cup X \cup Y$ in a triple in $M$ and one for each triple in $M$.

$\circ$ Let $W=\left\{w_{1}, \ldots, w_{q}\right\}, X=\left\{x_{1}, \ldots, x_{q}\right\}$, and $Y=$ $\left\{y_{1}, \ldots, y_{q}\right\}$. Given any $z \in W \cup X \cup Y$, let $N(z)$ denote the number of triples in $M$ that contain $z$, and let $z[1], z[2], \ldots, z[N(z)]$ denote the elements in $A$ corresponding to $z$. Let $r=32 q$, and define

$$
\begin{array}{rlrl}
s\left(w_{i}[1]\right)=10 r^{4}+i r+1, & & 1 \leq i \leq q, \\
s\left(w_{i}[l]\right)=11 r^{4}+i r+1, & & 1 \leq i \leq q, 2 \leq l \leq N\left(w_{i}\right), \\
s\left(x_{j}[1]\right)=10 r^{4}+j r^{2}+2, & 1 \leq j \leq q, \\
s\left(x_{j}[l]\right)=11 r^{4}+j r^{2}+2, & 1 \leq j \leq q, 2 \leq l \leq N\left(x_{j}\right), \\
s\left(y_{k}[1]\right)=10 r^{4}+k r^{3}+4, & 1 \leq k \leq q, \\
s\left(y_{k}[l]\right)=8 r^{4}+k r^{3}+4, & 1 \leq k \leq q, 2 \leq l \leq N\left(y_{k}\right) .
\end{array}
$$

- Let $u_{l}$ denote the single element corresponding to a particular triple $m_{l}=\left(w_{i}, x_{j}, y_{k}\right) \in M$. For any such $u_{l}$, let $s\left(u_{l}\right)=10 r^{4}-k r^{3}-j r^{2}-i r+8$.

○ $B=40 r^{4}+15$. 
Note that $\max \{s(a) \mid a \in A\} \leq 2^{16}|A|^{4}$. This means 4-PARTITION is NP-complete in the strong sense Garey \& Johnson (1979). It can be checked that this reduction is injective.

(II) From 4-Partition to 3-Partition (cf. Theorem 4.4 in Garey \& Johnson (1979)):

The 3-PARTition problem is to decide, given a set $A$ of size $3 m$, a positive integer bound $B$, a positive integer size $s(a)$ for each $a \in A$ such that $B / 4<s(a)<B / 2$ and $\sum_{a \in A} s(a)=$ $m B$, whether $A$ can be partitioned into $m$ disjoint subsets $A_{1}, \ldots, A_{m}$, each of size three, such that, for each $1 \leq i \leq m$, $\sum_{a \in A_{i}} s(a)=B$. We denote an instance of 3-PARTITION by the tuple $(A, m, B, s)$.

The reduction in Garey \& Johnson (1979) maps an instance $(A, m, B, s)$ of 4-PARTition, with $|A|=4 m$ and $\max \{s(a) \mid a \in$ $A\} \leq 2^{16}|A|^{4}$, to the instance $\left(A^{\prime}, m^{\prime}, B^{\prime}, s^{\prime}\right)$ of 3-PARTition, where $A^{\prime}$ has $m^{\prime}=O\left(m^{2}\right)$ elements: one element $w_{i}$ for each element $a_{i}$ of $A$, two elements $u_{i, j}$ and $\bar{u}_{i, j}$ for each pair $\left(a_{i}, a_{j}\right)$ of elements from $A$, and $8 m^{2}-3 m$ filler elements $u_{k}^{*}$, $1 \leq k \leq 8 m^{2}-3 m$. Their sizes are:

$$
\begin{aligned}
s^{\prime}\left(w_{i}\right) & =4\left(5 B+s\left(a_{i}\right)\right)+1, \\
s^{\prime}\left(u_{i, j}\right) & =4\left(6 B-s\left(a_{i}\right)-s\left(a_{j}\right)\right)+2, \\
s^{\prime}\left(\bar{u}_{i, j}\right) & =4\left(5 B+s\left(a_{i}\right)+s\left(a_{j}\right)\right)+2, \\
s^{\prime}\left(u_{k}^{*}\right) & =20 B .
\end{aligned}
$$

We let $B^{\prime}=64 B+4$. It can be checked that this reduction is injective.

(III) From 3-Partition to Machine Flow, the decision version of the two-machine flow scheduling problem with unit processing times defined in Chapter 3 in $\mathrm{Yu}$ (1996) (where it is called F2UD'):

The MACHINE FLOW problem is to decide, given two machines M1 and M2, each of which can process at most one job at a time, and $n$ jobs $j, 1 \leq j \leq n$, where each job takes unit processing time and the job $j$ is assigned a delay $l_{j}$ 
that describes the minimum amount of time between the completion of the job $j$ on M1 and its start on M2, and a threshold $y$, whether there exists a feasible schedule of the jobs so that the last job is completed before time $y$.

The reduction in $\mathrm{Yu}(1996)$ from 3-PARTITION to MACHINE FLOW goes as follows. Without loss of generality, we consider a modified version of 3-PARTITION by multiplying the partition elements by $4 \mathrm{~m}$. Thus we are given a set of positive integers $A=\left\{a_{1}, \ldots, a_{3 m}\right\}$ and a positive integer $B$ such that (1) $B<a_{i}<2 B$ for all $i$, (2) $\sum_{j} a_{j}=4 m B$, (3) $a_{i}=0$ $(\bmod m)$ for all $i$, and $(4) 4 B=0(\bmod m)$. The problem is to decide if $A$ can be partitioned into $m$ disjoint 3-element subsets $A_{1}, \ldots, A_{m}$ such that $\sum_{a_{j} \in A_{i}} a_{j}=4 B$, for all $i$. An instance of this modified version of 3-PARTITION is mapped to an instance of MACHINE FLOW with delays (1) $l_{j}=a_{j}$ for $1 \leq j \leq 3 m,(2) l_{j}=0$ for $3 m+1 \leq j \leq 4 m B,(3) l_{j}=u+1$ for $4 m B+1 \leq j \leq m u$, where $u=4(m+1) B$, and (4) the threshold $y=n+4 m B+2$, where $n=m u$ is the total number of jobs. It can be checked that this reduction is injective.

(IV) From Machine Flow to RN3DM (Restricted Numerical 3-Dimensional Matching, cf. page 31 in $\mathrm{Yu}$ 1996):

The RN3DM problem is to decide, given a positive integer set $U=\left\{u_{1}, \ldots, u_{n}\right\}$ and a positive integer $e$ such that $\sum_{j=1}^{n} u_{j}+$ $n(n+1)=n e$, whether there exist two $n$-permutations $\lambda$ and $\mu$ such that $j+\lambda(j)+u_{\mu(j)}=e$ for $1 \leq j \leq n$. (It can be assumed that each $\left.u_{i}<e-1\right)$.

The reduction (Corollary 3 on page 32 in $\mathrm{Yu}$ 1996) maps an instance of MACHINE FLOW to that of RN3DM given by $u_{j}=l_{j}$ for $1 \leq j \leq n$, and $e=y$. We assume that the instance of MACHINE FLOW here arises in the reduction from 3-Partition to Machine Flow given in (III) above. This will ensure that $\sum_{j} u_{j}+n(n+1)=n e$ and each $u_{i}<e-1$, which we require for $(\mathrm{V})$ below to be injective. It can be checked that this reduction is injective.

(V) From RN3DM to RNMTS (Restricted Numerical Matching 
with Target Sums, cf. Brunetti et al. 2008):

The RNMTS problem is to decide, given positive integers $y_{1}, \ldots, y_{n}$ such that $2 \leq y_{1} \leq y_{2} \leq \cdots \leq y_{n} \leq 2 n$ and $\sum_{i} y_{i}=n(n+1)$, if there exist $n$-permutations $\sigma$ and $\pi$ such that $\sigma(k)+\pi(k)=y_{k}$ for $1 \leq k \leq n$.

RN3DM is mapped to RNMTS by letting $y_{j}=e-u_{j}$ and then reordering the $y_{j}$ as per their values. It can be checked that this reduction is injective.

(VI) From RNMTS to Permutation:

The Permutation problem (page 69 in Brunetti et al. 2008, where it is called Permutation $\left.\left(S_{3}\right)\right)$ is to decide, given nonnegative integers $z_{2}, \ldots, z_{2 n} \in\{0, \ldots, n\}$, whether there exists an $n \times n$ permutation matrix $P$ such that $\sum_{i, j: i+j=l} P_{i, j}=z_{l}$ for $2 \leq l \leq 2 n$.

The reduction in Brunetti et al. (2008) maps an instance $y=\left(y_{1}, \ldots, y_{n}\right)$ of RNMTS to an instance $z=\left(z_{2}, \ldots, z_{2 n}\right)$ of Permutation by setting $z_{l}=\left|\left\{k \leq n \mid y_{k}=l\right\}\right|$. It can be checked that this reduction is injective.

(VII) From Permutation to Special Consistency:

Special Consistency is the problem addressed in Theorem 3.7, namely, the problem of deciding positivity of $t_{\mu, \pi}^{\lambda}$, given $\lambda, \mu$ and $\pi$ in unary, when $\left(\lambda^{T}, \mu^{T}, \pi^{T}\right)$ is restricted to be of the form (3.6).

The reduction in Brunetti et al. (2001) maps an instance $z=\left(z_{2}, \ldots, z_{2 n}\right)$ of PERMUTATION to an instance $(\lambda, \mu, \pi)$ of SpeCial Consistency satisfying (3.6), with $r=n-1$ and $d_{i}=z_{i+2}, 0 \leq i \leq 2 r$ (it can be shown that $\sum_{k} d_{k}=r+1$, and $\left.\sum_{k} k d_{k}=r(r+1)\right)$. This reduction is injective.

(VIII) From Special Consistency to Restricted Kronecker: The reduction given in the proof of Theorem 4.2 is also injective. 
Theorem 1.7 follows from Lemma 5.1 and Theorem 5.2. This proof also shows that the superpolynomially many partition triples in Theorem 1.7 can be constructed explicitly (as defined in Section 1.5).

REMARK 5.3. In the preceding proof, we can use, in place of 3D Matching, any problem in NP which has a polynomial-timecomputable padding function (Berman \& Hartmanis 1977), and which can be reduced by a polynomial-time one-one reduction to REStricted Kronecker. For example, SAT also has a polynomialtime-computable padding function, and it can also be reduced by a polynomial-time one-one reduction to RESTRICTED KRONECKER. This reduction is obtained by composing the injective reduction from Sat to 3D Matching given in Garey \& Johnson (1979) with the injective reduction from 3D MATCHING to RESTRICTED KRONECKER given in the proof of Theorem 5.2.

EXAMPLE 5.4. Though the reduction $\phi$ in Theorem 5.2 is polynomial-time computable, the blow-up in size can be substantial. For example, let us start with a trivial instance of the 3D MATCHING problem, wherein $q=2, W=\left\{w_{1}, w_{2}\right\}, X=\left\{x_{1}, x_{2}\right\}, Y=\left\{y_{1}, y_{2}\right\}$, and $M=\left\{\left(w_{1}, x_{1}, y_{1}\right),\left(w_{2}, x_{1}, y_{2}\right),\left(w_{1}, x_{2}, y_{2}\right)\right\}$. Clearly, $M$ does not contain a matching.

It can be checked that $\phi(M, W, X, Y, q)$, with $\epsilon=1$ in condition Theorem 1.7(iii), is a partition triple whose height is $>10^{16}$ and the total size is $>10^{46}$. By Theorem 5.2, the Kronecker coefficient associated with this partition triple is zero. One cannot verify this fact directly using a computer, since computation of Kronecker coefficients for partition triples of this height and size is far beyond the reach of computer algebra systems. Thus Theorem 5.2 maps instances of 3D MATCHING which do not contain matching for trivial reasons to partition triples whose associated Kronecker coefficients vanish for highly nontrivial reasons. Thus the image of something trivial is highly nontrivial. This happens because of the nontriviality of the sequence of reductions that produce the image. 
5.2. Proof of Theorem 1.8. For the proof of Theorem 1.8, we need the following lemma, which proves a variant of the result in Fortune (1979) that coNP-complete languages cannot be sparse unless $\mathrm{P}=\mathrm{NP}$.

LEMMA 5.5. Let $\mathcal{L}$ be a coNP-hard language given as a disjoint union

$$
\mathcal{L}=\mathcal{L}^{\prime} \cup \mathcal{L}^{\prime \prime}
$$

where $\mathcal{L}^{\prime}$ is sparse (i.e., there are only poly $(n)$ words of length $n$ in $\left.\mathcal{L}^{\prime}\right)$ and $\mathcal{L}^{\prime \prime} \in N P \cap \operatorname{coNP}$. Then coNP $=N P$.

ProOF. We will show that the assumptions imply that $\mathrm{SAT}^{c}$ (the complement of SAT) is in NP - this would imply that coNP $\subseteq \mathrm{NP}$, and hence, coNP $=$ NP. For this, we adapt the proof in Fortune (1979); Mahaney (1982).

Since $\mathcal{L}$ is coNP-hard, there exists a polynomial-time many-one reduction $R$ such that $R(\mathrm{SAT}) \subseteq \mathcal{L}^{c}$ and $R\left(\mathrm{SAT}^{c}\right) \subseteq \mathcal{L}$. Since $\mathcal{L}^{\prime \prime}$ is in NP $\cap$ coNP, there exist non-deterministic Turing machines $M_{1}$ and $M_{2}$ such that, given input $x, M_{1}$ halts (in polynomial time) if and only if $x \in \mathcal{L}^{\prime \prime}$, while $M_{2}$ halts (in polynomial time) if and only if $x \notin \mathcal{L}^{\prime \prime}$.

Let $F$ be a formula for which we have to decide unsatisfiability. We perform depth-first search on the binary tree obtained by self-reducing $F$ (the root of this tree is $F$, and the children of a node $G$ are $G_{0}$ and $G_{1}$, the formulas of smaller size obtained by specializing the first variable in $G$ to true or false, and applying trivial simplifications), starting at the root node. We maintain a table $\mathcal{U}$ of labels ( $R$-values) of unsatisfiable formulae, starting with $\mathcal{U}:=\{R$ (false) $\}$. At each node $G$, we first compute $R(G)$ and then do one of the following:

1. If $R(G) \in \mathcal{U}$, prune the subtree and return to the parent node.

2. Otherwise, if $G=$ true, enter an infinite loop.

3. Otherwise, run both non-deterministic Turing machines $M_{1}$ and $M_{2}$ in parallel on the input $R(G)$ until one of the two 
halts (which will always happen, for some sequence of nondeterministic choices, in polynomial time):

(a) If $M_{1}$ halts (in which case $R(G) \in \mathcal{L}^{\prime \prime} \subseteq \mathcal{L}$, and hence, $G$ is unsatisfiable), add $R(G)$ to $\mathcal{U}$, prune the subtree and return to the parent node.

(b) If $M_{2}$ halts, visit both children $G_{0}$ and $G_{1}$. Upon return (if this happens), it will always be true that $G_{0}$ and $G_{1}$ are unsatisfiable, and hence $R\left(G_{0}\right), R\left(G_{1}\right) \in \mathcal{U}$ and $G$ is unsatisfiable. Thus add $R(G)$ to $\mathcal{U}$ and return to the parent node.

It is clear that this algorithm can be understood as a non-deterministic Turing machine that halts if and only if $F$ is unsatisfiable.

It suffices to show that, if $F$ is unsatisfiable, this algorithm halts in polynomial time. For this, it suffices to show that the number of interior nodes that are visited by the algorithm is polynomial in the size $|F|$ of the formula $F$ (since the tree is binary, the number of visited leaves is at most twice the number of visited interior nodes). Now observe that interior nodes only arise in the case where $M_{2}$ halts on input $R(G)$, in which case $R(G) \in \mathcal{L}^{\prime}$. Thus any interior node is necessarily labeled by an element of the sparse set $\mathcal{L}^{\prime}$. We can thus conclude the argument precisely as in Lemma 2.2 of Mahaney (1982): If $G$ and $G^{\prime}$ are two interior nodes that have the same label, $R(G)=R\left(G^{\prime}\right) \in \mathcal{L}^{\prime}$, then they necessarily ought to appear in the same branch of the search tree (because we proceed by depth-first search). As the depth of the tree is no more than $m$ - the number of variables in $F$ - we find that each label can occur at most $m$ times. Therefore, the number of visited interior nodes can be upper bounded by $m \cdot p(q(|F|))$, where $q$ is a polynomial that bounds the increase in length induced by the reduction $R$ and $p=p(n)$ is a polynomial that bounds the number of strings of length $\leq n$ in the sparse set $\mathcal{L}^{\prime}$. We conclude that $\mathrm{SAT}^{c} \in \mathrm{NP}$.

Another ingredient needed for the proof of Theorem 1.8 is the following result.

TheOREM 5.6 (Bürgisser et al. 2017). The problem of deciding if

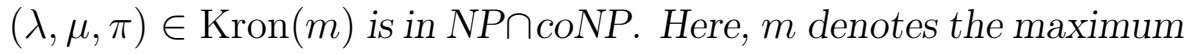


height of $\lambda, \mu$, or $\pi$, and the partition triple $(\lambda, \mu, \pi)$ is given in unary.

We can now give the proof of Theorem 1.8.

Proof (Theorem 1.8). For given $m$, let $\mathcal{L}$ be the set of partition triples $(\lambda, \mu, \mu)$ satisfying the constraints Theorem 1.7(i) (vi). Let $\mathcal{L}^{\prime}$ be the set of partition triples $(\lambda, \mu, \mu)$ satisfying both the constraints Theorem 1.7(i) (vi) and Theorem 1.8(vii) (viii), Let $\mathcal{L}^{\prime \prime}$ be the set of partition triples satisfying the constraints Theorem 1.7(i) (vi) such that either (vii) or (viii) in Theorem 1.8 are violated. Then, clearly, $\mathcal{L}=\mathcal{L}^{\prime} \cup \mathcal{L}^{\prime \prime}$.

In the definition of $\mathcal{L}^{\prime \prime}$, we can drop the constraint (i), since it is automatically satisfied if (vii) or (viii) are violated (as $k_{\mu, \mu}^{\lambda}=$ $k_{\mu^{t}, \mu}^{\lambda^{t}}=k_{\mu^{t}, \mu^{t}}^{\lambda}$ ). By Theorem 5.6, the problem of deciding whether a partition triple belongs to the Kronecker cone is in NP $\cap$ coNP. It follows that $\mathcal{L}^{\prime \prime} \in \mathrm{NP} \cap$ coNP. By Theorem 4.2, $\mathcal{L}$ is coNP-hard. It now follows from Lemma 5.5 that $\mathcal{L}^{\prime}$ is not sparse, assuming coNP $\neq$ NP. This proves Theorem 1.8 .

\section{Correlation between the complexity of $k_{\mu, \pi}^{\lambda}$ and $t_{\mu, \pi}^{\lambda}$}

There seems to be a surprising correlation between the complexities of $k_{\mu, \pi}^{\lambda}$ and $t_{\mu, \pi}^{\lambda}$. On the one hand, positivity of $k_{\mu, \pi}^{\lambda}$ is, in general, NP-hard to decide (Theorem 3.8, just as it is for $t_{\mu, \pi}^{\lambda}$ (Theorem 3.7). On the other hand, suppose $\Pi$ is a subclass of partition triples such that the problem of deciding positivity of $t_{\mu, \pi}^{\lambda}$, for $(\lambda, \mu, \pi) \in \Pi$, is in $P$. While the corresponding problem of deciding positivity of $k_{\mu, \pi}^{\lambda}$, for $(\lambda, \mu, \pi) \in \Pi$, may not always be in $P$, the results in this section suggest that it may indeed be so for many "natural" subclasses $\Pi$. In particular, Theorem 6.9 proved in this section suggests that the problem of deciding positivity of $k_{\mu, \pi}^{\lambda}$, when $\mu$ and $\pi$ are rectangular $(=\delta(\lambda))$, is in $P$, as conjectured in Mulmuley (2010b).

6.1. Interpretation of the $t$-function in terms of hypergraphs. We begin with a lemma that is needed for proving these 
results.

Definition 6.1. Let $d \in \mathbb{N}$. An obstruction predesign is a hypergraph with $d$ indistinguishable vertices and with hyperedges that come in three colors, such that every vertex lies in exactly one hyperedge of each color. The set all hyperedges that have the same color is called a layer of hyperedges, so in our case we have three layers of hyperedges.

Let $\lambda, \mu, \pi$ be partitions of $d$. An obstruction predesign is defined to have type $(\lambda, \mu, \pi)$ if the number of columns in $\lambda$ of length $k$ equals the number of hyperedges in layer 1 with $k$ vertices, and the number of columns in $\mu$ of length $k$ equals the number of hyperedges in layer 2 with $k$ vertices, and the number of columns in $\pi$ of length $k$ equals the number of hyperedges in layer 3 with $k$ vertices.

To each vertex we can assign its triple of hyperedges. An obstruction design is an obstruction predesign such that no two vertices have the same triple of hyperedges.

LEMMA 6.2. Let $\tilde{t}_{\mu, \pi}^{\lambda}$ be the number of obstruction designs of type $(\lambda, \mu, \pi)$. Then $t_{\mu, \pi}^{\lambda}>0$ iff $\tilde{t}_{\mu, \pi}^{\lambda}>0$.

ProOF. Recall from Section 2 that $t_{\mu, \pi}^{\lambda}$ is the number of point sets $P \subseteq \mathbb{N}^{3}$ with marginals $\left(\lambda^{T}, \mu^{T}, \pi^{T}\right)$. We define the $k$-th slice of a point set in direction $i, 1 \leq i \leq 3$, to be its subset consisting of the points that have $k$ as their $i$-th coordinate. (Here the directions 1,2 , and 3 correspond to the $x, y$, and $z$ coordinates, respectively.) We now prove that $t_{\mu, \pi}^{\lambda}>0$ iff $\tilde{t}_{\mu, \pi}^{\lambda}>0$. From a point set $P$ with marginals $\left(\lambda^{T}, \mu^{T}, \pi^{T}\right)$, we can define an obstruction design of type $(\lambda, \mu, \pi)$ by taking $P$ to be the hypergraph vertex set and making each slice in direction $i$ a hyperedge in layer $i$. Conversely, from an obstruction design of type $(\lambda, \mu, \pi)$ we can obtain a point set with marginals $\left(\lambda^{T}, \mu^{T}, \pi^{T}\right)$ as follows: For each layer we give consecutive numbers (starting at 0 ) to each hyperedge, beginning with the largest hyperedge and continuing in a manner such that the hyperedge sizes form a nonincreasing sequence, i.e., a partition of $d$. Since every vertex lies in exactly one hyperedge of each layer, every vertex gives rise to a triple of nonnegative integers, and the triples 
are pairwise distinct because no two vertices share all 3 hyperedges. These triples form a point set with marginals $\left(\lambda^{T}, \mu^{T}, \pi^{T}\right)$.

Note that $t_{\mu, \pi}^{\lambda}$ needs not always be equal to $\tilde{t}_{\mu, \pi}^{\lambda} ;$ this can happen when the hyperedge sizes are not all distinct. Though, by the preceding result, the problems of deciding positivity of $t_{\mu, \pi}^{\lambda}$ and $\tilde{t}_{\mu, \pi}^{\lambda}$ are equivalent, obstruction designs introduced here will turn out to be convenient in the proofs that follow.

6.2. Littlewood-Richardson coefficients. Given partitions $\lambda$, $\mu, \pi$ such that $|\lambda|=|\mu|+|\pi|$, let $\iota:=|\lambda|+\lambda_{1}$. Let $\tilde{\lambda}, \tilde{\mu}, \tilde{\pi}$ be the partitions $\lambda, \mu, \pi$ with a long first row put on top of their Young diagrams such that $|\tilde{\lambda}|=|\tilde{\mu}|=|\tilde{\pi}|=3 \iota$. Then, $k_{\tilde{\mu}, \tilde{\pi}}^{\tilde{\pi}}$ is equal to the Littlewood-Richardson coefficient $c_{\mu, \pi}^{\lambda}$ associated with the partition triple $(\lambda, \mu, \pi)$ (Murnaghan 1938).

The problem of deciding positivity of $k_{\tilde{\mu}, \tilde{\pi}}^{\tilde{\lambda}}=c_{\mu, \pi}^{\lambda}$ has a strongly polynomial time algorithm (Knutson \& Tao 2001; Mulmuley et al. 2011). This is consistent with the following result.

TheOREM 6.3. For any $\lambda, \mu$, and $\pi, t_{\tilde{\mu}, \tilde{\pi}}^{\tilde{\pi}}>0$. In particular, the problem of deciding positivity of $t_{\tilde{\mu}, \tilde{\pi}}^{\tilde{\pi}}$ is trivial.

ProOF. By Lemma 6.2, it suffices to construct a hypergraph to show that $\tilde{t}_{\tilde{\mu}, \tilde{\pi}}^{\tilde{\lambda}}>0$. We call a hyperedge that contains only a single vertex a singleton. The key property of the constructed hypergraph will be that every vertex lies in 2 singletons and another hyperedge. By construction $\tilde{\lambda}, \tilde{\mu}$, and $\tilde{\pi}$ each have at least $2 \iota$ columns with a single box. We split the $3 \iota$ vertices into three equally sized parts. The vertices of the first part are contained in singleton hyperedges of layer 2 and layer 3 . The vertices of the second part are contained in singleton hyperedges of layer 1 and layer 3 . The vertices of the third part are contained in singleton hyperedges of layer 1 and layer 2 . The remaining hyperedges of layer $i$ are constructed by freely partitioning the vertices in the $i$-th part according to the desired hyperedge sizes. Since no two vertices share all three hyperedges, the theorem is proved.

6.3. Partitions of constant height. It is known that positivity of $k_{\mu, \pi}^{\lambda}$ can be decided in polynomial time when $\lambda, \mu$, and $\pi$ have 
constant heights. This is consistent with the following result.

TheOrem 6.4. Fix a constant $c \in \mathbb{N}$. If $|\lambda|=|\mu|=|\pi|$ and $\operatorname{ht}(\lambda) \leq c, \operatorname{ht}(\mu) \leq c, \operatorname{ht}(\pi) \leq c$, then positivity of $t_{\mu, \pi}^{\lambda}>0$ can be decided in polynomial time.

PROOF. The algorithm is a hybrid algorithm based on the number of boxes $|\lambda|$. The values for $t$ in the case $|\lambda|<(c+2) c$ are stored in a database of constant size. The case $|\lambda| \geq(c+2) c$ is trivial, as the following lemma shows.

LEMMA 6.5. If ht $(\lambda) \leq c, \operatorname{ht}(\mu) \leq c, \operatorname{ht}(\pi) \leq c$, and $|\lambda|=|\mu|=$ $|\pi| \geq(c+2) c$, then $t_{\mu, \pi}^{\lambda}>0$.

ProOF. By Lemma 6.2, it suffices to construct a hypergraph showing that $\tilde{t}_{\mu, \pi}^{\lambda}>0$.

If $|\lambda|$ is divisible by $c$, then we arrange the vertices in a rectangular array whose columns contain $c$ vertices each. Otherwise we add an extra column containing less than $c$ vertices. The crucial property is that since $|\lambda| \geq(c+2) c$ each row contains at least $c+2$ vertices.

Proceeding column-wise from top to bottom and from left to right, we greedily assign vertices to hyperedges of the first layer according to the column lengths of $\mu$. Note that each hyperedge constructed thus lies either in a single column or in two adjacent columns. Likewise, proceeding row-wise from left to right and from top to bottom, we greedily assign vertices to hyperedges of the second layer according to the column lengths of $\pi$. Since each row contains at least $c+2$ vertices, a layer 2 hyperedge cannot contain two vertices from the same or adjacent columns.

Consider two vertices $v$ and $w$ that lie in the same layer 1 hyperedge. They either lie in the same column or in adjacent columns. Therefore no layer 2 hyperedge can contain both $v$ and $w$. This shows that we can choose an arbitrary layer 3 hyperedge arrangement and see that $\tilde{t}_{\mu, \pi}^{\lambda}>0$.

Note that $t_{\mu, \pi}^{\lambda}>0$ and $t_{\mu^{\prime}, \pi^{\prime}}^{\lambda^{\prime}}>0$ implies $t_{\mu+\mu^{\prime}, \pi+\pi^{\prime}}^{\lambda+\lambda^{\prime}}>0$, and that Lemma 6.5 shows that for constant height the semigroup of triples 
with positive $t_{\mu, \pi}^{\lambda}$ is finitely generated, as it is known for $k_{\mu, \pi}^{\lambda}$ as well.

6.4. When one partition is a hook. Blasiak (2017) has a given a \#P-formula for $k_{\mu, \pi}^{\lambda}$ when $\lambda$ is a hook. The problem of deciding positivity of $k_{\mu, \pi}^{\lambda}$ in this case may be conjectured to be in $P$ in view of the following result.

Theorem 6.6. Positivity of $t_{\mu, \pi}^{\lambda}$, given $\lambda, \mu$, and $\pi$ in unary, can be decided in polynomial time if $\lambda$ is a hook.

We give the proof of Theorem 6.6 in the remainder of this subsection. Let $\lambda=\left(D-k+1,1^{k-1}\right)$ be a hook partition with $D$ boxes. The Young diagram $\lambda$ has $D-k$ columns that contain only a single box and a single column with $k$ boxes. Let $\mu$ and $\pi$ be arbitrary partitions of $D$. By Lemma 6.2, it suffices to decide in polynomial time whether $\tilde{t}_{\mu, \pi}^{\lambda}>0$, i.e., if an obstruction design of type $(\lambda, \mu, \pi)$ exists.

The first key observation is the following. If we fix all the hyperedges in the $\mu$ and $\pi$ layers and ask whether an obstruction design of type $(\lambda, \mu, \pi)$ exists with these prescribed hyperedges, then this is easy to answer: For fixed set partitions $\alpha$ and $\beta$ that have hyperedge sizes $\mu^{T}$ and $\pi^{T}$, respectively, let $\tilde{t}_{\mu, \pi}^{\lambda}(\alpha, \beta)$ denote the number of obstruction designs whose $\mu$ layer is $\alpha$ and whose $\pi$ layer is $\beta$. We call two vertices that lie in the same $\mu$-hyperedge and the same $\pi$-hyperedge $(\alpha, \beta)$-equivalent.

ClaIm 6.7. $\tilde{t}_{\mu, \pi}^{\lambda}(\alpha, \beta)=0$ iff $k$ is larger than the number of $(\alpha, \beta)$ equivalence classes.

Proof. Fix $\alpha$ and $\beta$. If $k$ is larger than the number of $(\alpha, \beta)$ equivalence classes, then by the pigeonhole principle the $\lambda$-hyperedge of size $k$ must contain two $(\alpha, \beta)$-equivalent vertices. Therefore this construction does not yield an obstruction design. If $k$ is smaller than the number of $(\alpha, \beta)$-equivalence classes, the $\lambda$-hyperedge of size $k$ can be chosen to contain pairwise $(\alpha, \beta)$-nonequivalent vertices. The other vertices are singletons in the $\lambda$ layer, so this construction yields an obstruction design. 
Thus being able to answer the question whether $\tilde{t}_{\mu, \pi}^{\lambda}>0$ is equivalent to answering the following question: Given hyperedge size vectors $\mu^{T}$ and $\pi^{T}$, what is the maximal number of $(\alpha, \beta)$ equivalence classes, where $\alpha$ and $\beta$ have hyperedge sizes $\mu^{T}$ and $\pi^{T}$, respectively? We will formalize this as a max flow problem with integer edge capacities (given in unary). Such a problem can be solved in polynomial time using the Ford-Fulkerson algorithm. We construct a directed graph with a source vertex, one vertex for each column of $\mu$, one vertex for each column of $\pi$, and one sink vertex. There are edges from the source vertex to the $\mu$-vertices whose capacity equals the number of boxes in the corresponding column of $\mu$. Analogously there are edges from the $\pi$-vertices to the sink vertex whose capacity equals the number of boxes in the corresponding column of $\pi$. Moreover, there is a capacity 1 edge from every $\mu-$ vertex to every $\pi$-vertex. The Ford-Fulkerson algorithm finds in polynomial time an integer solution to the problem of sending the maximum amount of flow through this network with respect to the capacity constraints. Combined with Claim 6.7 the following claim implies that the Ford-Fulkerson algorithm can be used to decide in polynomial time whether $\tilde{t}_{\mu, \pi}^{\lambda}>0$ is positive.

Claim 6.8. A solution with flow at least $k$ exists iff there exist $\alpha$ and $\beta$ such that the number of $(\alpha, \beta)$-equivalence classes is at least $k$.

Proof. Given $\alpha$ and $\beta$ with at least $k$ equivalence classes we construct a solution to the flow problem by sending one flow unit for each equivalence class: For the equivalence class corresponding to the $i$ th $\mu$-column and $j$ th $\pi$-column we send a unit from the source vertex to the $i$ th $\mu$-vertex, from there to the $j$ th $\pi$-vertex and then to the sink vertex. This satisfies the capacity constraints and is a solution to the flow problem that sends at least $k$ flow units.

From a solution of the max flow problem we readily generate a solution to a relaxed max flow problem where we remove the capacities on the edges from the $\mu$-vertices to the $\pi$-vertices. We send flow units on additional arbitrary paths from the source to the sink. Once all capacities are saturated we are guaranteed to 
send exactly $D$ flow units. From this new solution we construct set partitions $\alpha$ and $\beta$ by defining that the size of the $(\alpha, \beta)$-equivalence class corresponding to the $i$ th $\mu$-column and the $j$ th $\pi$-column is the amount of flow from the $i$ th $\mu$-vertex to the $j$ th $\pi$-vertex. So if the original solution had at least $k$ flow units, then there are at least $k(\alpha, \beta)$-equivalence classes in our construction.

6.5. Rectangular Kronecker coefficients. It is conjectured in Mulmuley (2010b) that the problem deciding positivity of the rectangular Kronecker coefficient $k_{\delta(\lambda), \delta(\lambda)}^{\lambda}$ is in $P$. This is supported by the following result.

TheOREm 6.9. Let $\lambda$ be any partition with $d r$ boxes and at most $\min \left(d^{2}, r^{2}\right)$ rows, and let $\delta=\delta(\lambda)=(d, \ldots, d)$ ( $r$ times). Then $t_{\delta, \delta}^{\lambda}>0$. In particular, the problem of deciding positivity of $t_{\delta, \delta}^{\lambda}$ is trivial.

Since $k_{\delta, \delta}^{\lambda}=0$ if $h t(\lambda)>\min \left(d^{2}, r^{2}\right)$, the constraint on $\lambda$ here is very natural.

ProOF. By Lemma 6.2, it suffices to prove positivity of $\tilde{t}_{\delta, \delta}^{\lambda}$. We do this by an explicit construction.

The case $d \geq r$ is easier, so we handle this case first. We have to construct an obstruction design with $d r$ vertices and go about it as follows. Let $i$ rem $d$ denote the remainder when dividing $i$ by $d$. The vertex set $V$ is a subset of the $d \times d$ grid $\{(i, j) \mid 0 \leq i, j<d\}$. We have $(i, j) \in V$ iff $(i+j)$ rem $d \in\{0,1, \ldots, r-1\}$. For example, for $r=4$ and $d=6$, the vertex set is arranged as follows (row 0 is at the top, column 0 is at the left):

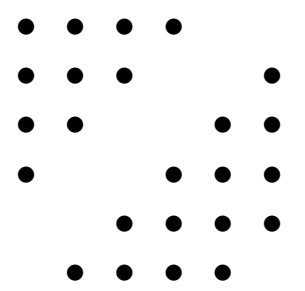

Note that every row and every column has exactly $r$ boxes. The rows correspond to the hyperedges of the first layer, where the columns correspond to the hyperedges of the second layer. Note 
that no matter how the hyperedges of the third layer are placed, no two vertices can share all three hyperedges, because no two vertices even share their two hyperedges in layer one and two. Therefore an arbitrary placement of the third layer shows $t_{\delta, \delta}^{\lambda}>0$.

For $d<r$ an analogous construction can be made, but several vertices share a location, see the example $r=6$ and $d=4$ below.

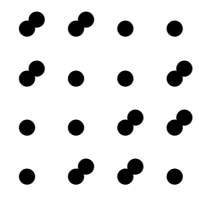

Note that in this construction, if $r / d>2$, then three or more vertices lie at the same position. As in the case $d \geq r$, the rows correspond to the hyperedges of the first layer, where the columns correspond to the hyperedges of the second layer. But now the third layer cannot be placed arbitrarily, but care has to be taken. The hyperedges can be placed in any order, but not at arbitrary positions. When a hyperedge is placed, it first uses those places where several vertices are grouped together (and of course only uses one from each such place). If there are places with more than two vertices, the hyperedge first takes vertices from those places with the most vertices. This greedy method ensures that no hyperedge contains a pair of vertices from the same place, because by the length restriction on $\lambda$ a hyperedge cannot use more than $d^{2}$ vertices.

\section{Acknowledgements}

This work was supported by NSF grant CCF-1017760. MW acknowledges support by the Simons Foundation, FQXI, and AFOSR (grant no. FA9550-16-1-0082). A part of this work was done at the Simons Institute for the Theory of Computing, Berkeley.

\section{References}

V. Baldoni, M. Vergne \& M. Walter (2017). Computation of Dilated Kronecker Coefficients. To appear in Journal of Symbolic Computation . 
A. Berenstein \& R. Sjamaar (2000). Coadjoint orbits, moment polytopes, and the Hilbert-Mumford criterion. Journal of the AMS 13(2), 433-466.

L. Berman \& J. Hartmanis (1977). On isomorphisms and density of NP and other complete sets. SIAM Journal on Computing 6, 305-322.

J. Blasiak (2017). Kronecker coefficients for one hook shape. Seminaire Lotharingien de Combinatoire $\mathbf{7 7}$.

J. Blasiak, K. Mulmuley \& M. Sohoni (2015). Geometric complexity theory IV: nonstandard quantum group for the Kronecker problem. Memoirs of the AMS 235(1109).

S. Brunetti, A. Del Lungo \& Y. Gerard (2001). On the computational complexity of reconstructing three-dimensional lattice sets from their two-dimensional X-rays. Linear Algebra and its Applications 339(1), 59-73.

S. Brunetti, A. Del Lungo, P. Gritzmann \& S. De Vries (2008). On the reconstruction of binary and permutation matrices under (binary) tomographic constraints. Theoretical Computer Science 406, 63-71.

P. Bürgisser, M. Christandl \& C. Ikenmeyer (2011a). Nonvanishing of Kronecker coefficients for rectangular shapes. Advances in Mathematics 227(5), 2082-2091.

P. Bürgisser, M. Christandl, K. Mulmuley \& M. Walter (2017). Membership in Moment Polytopes is in NP and coNP. SIAM Journal on Computing 46(3), 972-991.

P. Bürgisser \& C. Ikenmeyer (2013). Explicit lower bounds via geometric complexity theory. In Proceedings of the 44th STOC, 141-150. ACM.

P. Bürgisser, J. Landsberg, L. Manivel \& J. Weyman (2011b). An overview of mathematical issues arising in the geometric complexity theory approach to $V P \neq V N P$. SIAM Journal on Computing 40(4), 1179-1209.

Peter BÜrgisser (2016). Permanent versus determinant, obstructions, and Kronecker coefficients. Seminaire Lotharingien de Combinatoire $\mathbf{7 5}$. 
M. Christandl, B. Doran \& M. Walter (2012). Computing Multiplicities of Lie Group Representations. In Proceedings of the 53rd FOCS, 639-648.

H. Derksen \& J. Weyman (2000). Semi-invariants of quivers and saturation for Littlewood-Richardson coefficients. Journal of Applied and Computational Mathematics 13(3), 467-479.

S. Fortune (1979). A note on sparse complete sets. SIAM Journal on Computing 8(3), 431-433.

W. Fulton \& J. Harris (1991). Representation theory: A first course. Springer.

M. Garey \& D. Johnson (1979). Computers and Intractability. W. H. Freeman and company, New York.

C. Ikenmeyer (2012). Geometric Complexity Theory, Tensor Rank, and Littlewood-Richardson Coefficients. Ph.D. thesis, Institute of Mathematics, University of Paderborn. Online available at http://nbn-resolving de/urn:nbn:de:hbz:466:2-10472.

Christian Ikenmeyer \& Greta Panova (2016). Rectangular Kronecker coefficients and plethysms in geometric complexity theory. In Proceedings of the 57th FOCS, 396-405. IEEE.

H. Kadish \& J. LAndsberg (2014). Padded polynomials, their cousins, and geometric complexity theory. Communications in Algebra 42(5), 2171-2180.

R. KARP (1972). Reducibility among combinatorial problems. Complexity of Computer Computations 85-103.

F. KIRWAN (1984). Convexity properties of the moment mapping, III. Invent. Math. 77, 547-552.

A. KlyachKo (2004). Quantum marginal problem and representations of the symmetric group. arXiv:quant-ph/0409113 .

A. Knutson \& T. TaO (1999). The Honeycomb model of $G L_{n}(\mathbb{C})$ tensor products I: proof of the saturation conjecture. Journal of the AMS 12, 1055-1090. 
A. Knutson \& T. TAO (2001). Honeycombs and sums of Hermitian matrices. Notices of the AMS 48, 175-186.

S. Kumar (2015). A study of the representations supported by the orbit closure of the determinant. Compositio Mathematica 151, 292-312.

S. Mahaney (1982). Sparse complete sets for NP: Solution of a conjecture by Berman and Hartmanis. Journal of Computer and System Sciences 25, 130-143.

L. Manivel (1997). Applications de Gauss et pléthysme. In Annales de l'institut Fourier, volume 47, 715-774. Chartres: L'Institut, 1950-.

K. Mulmuley (2010a). Explicit proofs and the flip. arXiv:1009.0246 .

K. Mulmuley (2010b). Geometric complexity theory VI: the flip via positivity. Technical report, Computer Science Department, the University of Chicago .

K. Mulmuley (2011). On $P$ vs. NP and geometric complexity theory. Journal of the ACM 58(2).

K. Mulmuley, H. Narayanan \& M. Sohoni (2011). Geometric complexity theory III: on deciding nonvanishing of a Littlewood-Richardson coefficient. Journal of Algebraic Combinatorics 1-8.

K. Mulmuley \& M. Sohoni (2008). Geometric complexity theory II: towards explicit obstructions for embeddings among class varieties. SIAM Journal on Computing 38(3), 1175-1206.

F. Murnaghan (1938). The analysis of the Kronecker product of irreducible representations of the symmetric group. American Journal of Mathematics 60(3), 761-784.

N. Ressayre (2010). Geometric invariant theory and the generalized eigenvalue problem. Inventiones mathematicae 180, 389-441.

R. Stanley (2002). Positivity problems and conjectures in algebraic combinatorics. In Mathematics: Frontiers and Perspectives 295-319.

E. VAllejo (2000). Plane partitions and characters of the symmetric group. Journal of Algebraic Combinatorics 11(1), 79-88. 
Michèle Vergne \& Michael Walter (2017). Inequalities for Moment Cones of Finite-Dimensional Representations. To appear in Journal of Symplectic Geometry .

M. Walter (2014). Multipartite Quantum States and their Marginals. Ph.D. thesis, ETH Zurich. ArXiv:1410.6820.

W. YU (1996). The two-machine flow shop problem with delays and the one-machine total tardiness. Ph.D. thesis, Eindhoven University of Technology.

Manuscript received 8 March 2016

Christian Ikenmeyer

Department of Mathematics

Texas A\&M University

College Station, TX 77843, USA

cikenmey@mpi-inf .mpg. de

http://people.mpi-inf.mpg.de/

cikenmey/

Ketan D. Mulmuley

Computer Science Department

The University of Chicago

Chicago, IL 60637, USA

mulmuley@uchicago.edu

http://gct.cs.uchicago.edu
Current address of CHRISTIAN IKENMEYER:

Max Planck Institute for Informatics

Saarland Informatics Campus

66123 Saarbrücken, Germany

Michael Walter

Stanford Institute for Theoretical Physics

Stanford University

Stanford, CA, 94305, USA

michael.walter@stanford.edu

http://web.stanford.edu/ waltemic/ 